\title{
A re-evaluation of dust processing in supernova shock waves ${ }^{\star}$
}

\author{
Marco Bocchio ${ }^{1}$, Anthony P. Jones ${ }^{1}$, and Jonathan D. Slavin ${ }^{2}$ \\ ${ }^{1}$ Institut d'Astrophysique Spatiale (IAS), UMR 8617, CNRS/Université Paris-Sud, 91405 Orsay, France \\ e-mail: marco.bocchio@ias.u-psud.fr \\ 2 Harvard-Smithsonian Center for Astrophysics, MS 83, 60 Garden Street, Cambridge, MA 02138, USA
}

Received 11 June 2014 / Accepted 30 July 2014

\begin{abstract}
Context. There is a long-standing and large discrepancy between the timescale for dust formation around evolved stars and the rapid dust destruction timescale in interstellar shocks.

Aims. We use our latest estimates for dust processing to re-evaluate the dust destruction efficiency in supernova triggered shock waves, estimate the dust lifetime, and calculate the emission and extinction from shocked dust.

Methods. We modelled the sputtering and fragmentation of grains in interstellar shocks for shock velocities between $50 \mathrm{~km} \mathrm{~s}^{-1}$ and $200 \mathrm{~km} \mathrm{~s}^{-1}$. We constrained the dust destruction using our recent dust model. Finally, we coupled our code to the DustEM code in order to estimate the emission and extinction from the dust post-shock.

Results. Carbonaceous grains are quickly destroyed, even in a $50 \mathrm{~km} \mathrm{~s}^{-1}$ shock, leading to a shorter lifetime than in previous studies. Silicate grains appear to be more resilient, but the new destruction lifetime that we find is similar to previous studies and short compared to the dust injection timescale.

Conclusions. The calculated fraction of elements locked in grains is not compatible with the observed values and therefore implies the re-formation of dust in the dense regions of the interstellar medium. Better modelling of the silicate sputtering together with hydrodynamical simulations of interstellar shocks, appears to reduce the silicate destruction and may close the destruction-formation timescale gap.
\end{abstract}

Key words. ISM: abundances - dust, extinction - methods: numerical - shock waves

\section{Introduction}

It is observationally well established that shock waves in the interstellar medium (ISM) are able to destroy dust grains (Routly $\&$ Spitzer 1952; Cowie 1978; Welty et al. 2002). The most recent theoretical studies (e.g. Jones et al. 1994, 1996; Serra Díaz-Cano \& Jones 2008; Jones \& Nuth 2011) estimated the dust destruction in supernova (SN) shock waves, restricting their calculations to shocks in the warm ionised medium (WIM), where the dominant destruction occurs (Seab 1987; McKee 1989). They found a lifetime of $\sim 2 \times 10^{8} \mathrm{yr}$ for carbonaceous grains and $\sim 4 \times 10^{8} \mathrm{yr}$ for silicate grains. However, since the early studies of dust processing in shock waves (Barlow 1978a,b; Draine \& Salpeter 1979a,b; Dwek \& Scalo 1980; Seab \& Shull 1983; McKee et al. 1987; Jones et al. 1994, 1996), our dust modelling has evolved considerably, so it is timely to revisit this issue.

Dust is formed in cirmustellar regions (around AGB stars) and also in supernovae ( $\mathrm{SNe}$ ). The dust injection timescale from asymptotic giant branch (AGB) stars has been estimated to be $\sim 3 \times 10^{9}$ yr (Dwek \& Scalo 1980; Gehrz 1989; Jones \& Tielens 1994). On the other hand, the formation of dust in the ejecta of core collapse $\mathrm{SNe}$ is a debated topic. For example, observations of type II SNe, such as Cas A or Supernova 1987A, reveal the presence of newly formed dust $\left(\leqslant 0.1 M_{\odot}\right.$ for CasA, Arendt et al. 2014, 0.4-0.7 $M_{\odot}$ for Supernova 1987A, Matsuura et al. 2011). However, these $\mathrm{SNe}$ have not yet reached the phase where the reverse shock would destroy part of the observed dust. Furthermore, analyses of presolar dust grains within primitive

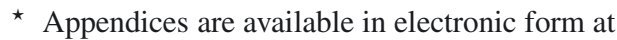
http://www . aanda.org meteorites seem to indicate $\mathrm{SiC}$ and graphite grains formed in Type II SNe (e.g. Lodders 2006; Hoppe et al. 2009, 2010). If a significant amount of dust was formed in $\mathrm{SN}$ ejecta, this could considerably reduce the dust injection timescale, but as yet the evidence is inconclusive.

Based on these results, the injection timescale is much longer than the theoretical lifetime of dust, and we would therefore expect to see a large fraction of heavy elements (i.e. C, Si, Fe, $\mathrm{Mg}, \mathrm{O}$, etc.) in the gas phase and almost nothing in dust grains. On the contrary, the observational evidence shows that most of the heavy elements are locked into grains (i.e. $\geqslant 50 \%$ for $\mathrm{C}$ and $\geqslant 90 \%$ for $\mathrm{Si}, \mathrm{Fe}$ and $\mathrm{Mg}$ ) in the diffuse ISM (Snow \& Witt 1996; Jenkins 2009 and references therein). This represents a longstanding conundrum and implies the re-formation of dust grains by accretion from the gas phase in denser regions of the ISM in order to match the observations. It has been shown that there are viable mechanisms for the re-formation of carbonaceous grains from the gas phase under low temperature and pressure conditions (e.g. Dartois et al. 2005). On the other hand, experimental and observational results seems to indicate that silicate grains are hard to form under ISM conditions and must therefore be formed in AGB stars and preserved in interstellar shocks (Jones \& Nuth 2011).

In this paper we re-evaluate the dust processing and lifetime using updated dust processing. We model the dust destruction in $\mathrm{SN}$ shock waves and the evolution of the size distribution of dust using a new version of the GRASH code used by Jones et al. (1994, 1996). We improve upon the erosion calculations by updating the carbonaceous material from graphite to hydrogenated amorphous carbon, use a molecular approach for the dissociation 
Table 1. DustEM parameters of the Jones et al. (2013) dust model.

\begin{tabular}{cccccccccc}
\hline \hline $\begin{array}{c}\text { Composition } \\
\text { core/mantle }\end{array}$ & $\begin{array}{c}\text { Size } \\
\text { distribution }\end{array}$ & $\begin{array}{c}E_{\mathrm{g}}(\mathrm{eV}) \\
\text { core/mantle }\end{array}$ & $\begin{array}{c}\rho\left(\mathrm{g} \mathrm{cm}^{-3}\right) \\
\text { core/mantle }\end{array}$ & $\alpha$ & $\begin{array}{c}a_{\min } / a_{\max } \\
(\mathrm{nm})\end{array}$ & $\begin{array}{c}a_{\mathrm{c}}, a_{\mathrm{t}} / a_{0} \\
(\mathrm{~nm})\end{array}$ & $\gamma / \sigma$ & $\begin{array}{c}Y \\
\left(M / M_{\mathrm{H}}\right)\end{array}$ & $f_{\mathrm{M} \text {-tot }}$ \\
\hline $\mathrm{a}-\mathrm{C}: \mathrm{H} / \mathrm{a}-\mathrm{C}$ & power-law & $2.5 / 0.1$ & $1.3 / 1.6$ & 5.0 & $0.4 / 4900$ & $50,10 /-$ & $1.0 /-$ & $1.6 \times 10^{-3}$ & $18.6 \%$ \\
a-C:H/a-C & log-normal & $2.5 / 0.1$ & $1.3 / 1.6$ & - & $0.5 / 4900$ &,$--/ 7.0$ & $-/ 1.0$ & $0.6 \times 10^{-3}$ & $7.0 \%$ \\
a-SilFe/a-C & log-normal & $\sim 8 / 0.1$ & $2.5 / 1.6$ & - & $1.0 / 4900$ &,$--/ 8.0$ & $-/ 1.0$ & $5.8 \times 10^{-3}$ & $67.4 \%$ \\
$-/ \mathrm{a}-\mathrm{C}$ & mantle & $-/ 0.1$ & $-/ 1.6$ & - & $\mathrm{d}=5 \mathrm{~nm}$ &,$--/-$ & $-/-$ & $0.6 \times 10^{-3}$ & $7.0 \%$ \\
\hline
\end{tabular}

Notes. $E_{\mathrm{g}}$ and $\rho$ are the band gap and the density of the material. The power-law distribution is defined as $\mathrm{d} n / \mathrm{d} a \propto a^{\alpha}$ and an exponential decay is applied by multiplying it by $\exp \left(-\left[\left(a-a_{\mathrm{t}}\right) / a_{\mathrm{c}}\right]^{\gamma}\right)$ for $a>a_{\mathrm{t}}$, while the $\log$-normal distribution is defined as $\mathrm{d} n / \mathrm{d} \log a \propto \exp \left(-\frac{\left(\log \left(a / a_{0}\right)\right)^{2}}{2 \sigma^{2}}\right)$.

of small carbonaceous grains, update the grain charge treatment by replacing the McKee et al. (1987) analytical charge scheme with the Weingartner \& Draine (2001) grain charge calculations and introduce a new and more physical dust model (i.e. Jones et al. 2013). Finally we couple the GRASH code to the DustEM code (Compiègne et al. 2011) in order to calculate the emission and extinction of shocked dust.

This paper is organised as follows. In Sect. 2 we summarise the main features of the Jones et al. (2013) dust model and in Sect. 3 we give an outline of the code presented by Jones et al. (1994, 1996) and present the new features introduced in the code. In Sects. 4 and 5 we present and discuss our results, and in Sect. 6 we summarise our conclusions.

\section{The Jones et al. (2013) dust model}

Recently, (Jones et al. 2013) has proposed a new interstellar dust model, which uses only two materials: (hydrogenated) amorphous carbon, a-C(:H), and forsterite-type silicate material with iron nano-inclusions $\left(\mathrm{a}-\mathrm{Sil} \mathrm{Fe}_{\mathrm{Fe}}\right)$. The dust size distribution consists of:

- a power law distribution of small $(a \simeq 0.4-100 \mathrm{~nm})$ aromatic-rich a-C grains;

- a log-normal distribution, centred at $a \sim 130 \mathrm{~nm}$, of large aliphatic-rich core/aromatic-rich mantle, a-C:H/a-C grains;

- a log-normal distribution, centred at $a \sim 160 \mathrm{~nm}$, of large amorphous silicate grains with $5 \mathrm{~nm}$ thick a-C mantle, a-Sil $\mathrm{Fe} / \mathrm{a}-\mathrm{C}$.

The a-C mantling of the silicate grains reflects the expectation that these two materials will inevitably mix via accretion in the ISM. Figure 1 shows the dust size distribution assumed in the model, compared to the MRN-like (Mathis et al. 1977) powerlaw grain size distribution adopted by Jones et al. (1994, 1996). The inner core of the carbonaceous grains is aliphatic-rich with an external, $20 \mathrm{~nm}$ thick layer of aromatic-rich material resulting from photo processing. In Table 1 we summarise the main parameters of the dust model as used in DustEM.

This dust model uses laboratory-based optical and physical properties for carbonaceous (Jones 2012a,b,c,d,e) and silicate grains (Jones et al. 2013). In particular, small carbonaceous grains follow a power law and are not divided into separate dust populations as in previous dust models (e.g. Draine \& Li 2001, 2007; Compiègne et al. 2011), their optical properties are continuous and size-dependent from bulk material down to $0.3 \mathrm{~nm}$, therefore making the characterisation of the grains more physical.

With the calculated optical properties, the dust model gives a good fit to the dust observables in the diffuse ISM. Furthermore,

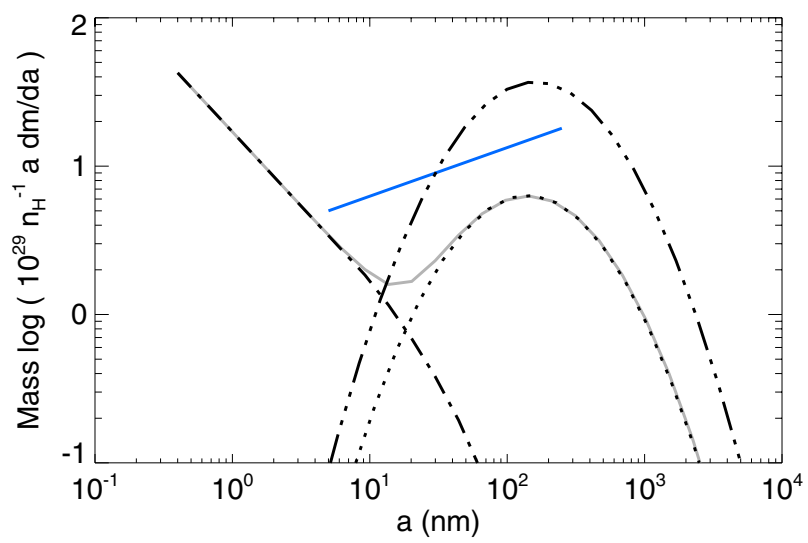

Fig. 1. Size distributions for the three dust populations in the Jones et al. (2013) dust model. Small and large carbonaceous grain size distributions (dot-dashed and dotted lines respectively). a-C coated silicate grains (triple-dot-dashed line). The grey line represents the overall carbonaceous grain size distribution, while the blue line is the power-law graphitic-type and silicate grain size distribution assumed by Jones et al. $(1994,1996)$. The assumed dust-to-gas mass ratio, $M / M_{\mathrm{H}}$, is the same for the two dust models.

with small variations in the physical and optical properties of carbonaceous grains, it is possible to describe the evolution of dust in a wide range of ISM environments (Jones 2013; Jones et al. 2014; Köhler, in prep.).

\section{The GRASH code}

GRASH is a FORTRAN code $^{1}$ that follows the processing of dust in supernova-generated interstellar shock waves. The methodology of the code was first presented by Jones et al. (1994). In the code they implemented the grain acceleration theory from McKee et al. (1987) and the treatment of destructive processes from Tielens et al. (1994). To guarantee the mass conservation throughout the shock, they modelled the mass loss processes using an algorithm based on the grain coagulation model of Nakagawa et al. (1981) and Mizuno et al. (1988). The processes taken into account were thermal and inertial sputtering and vapourisation. In 1996 shattering was added to the dust processing (Jones et al. 1996). They assumed graphitic-type carbon and silicate grains following an MRN-like size distribution. They did not co-solve the shock dynamics and grain physics but followed the dust evolution self consistently with the shock

1 The GRASH code was originally written and developed by A. P. Jones in the period 1990 to 1995 . 
Table 2. parameters for sputtering of graphitic-type and a-C:H grains.

\begin{tabular}{ccccccc}
\hline \hline Material & $\rho\left(\mathrm{g} \mathrm{cm}^{-3}\right)$ & $X_{\mathrm{H}}$ & $U_{0}(\mathrm{eV})$ & $K$ & $\left\langle Z_{2}\right\rangle$ & $\left\langle M_{2}\right\rangle$ \\
\hline graphite & 2.2 & 0 & 4 & 0.65 & 6.0 & 12 \\
a-C:H & 1.4 & 0.4 & 4 & -0.04 & 4.0 & 7.6 \\
\hline
\end{tabular}

structure profiles (Raymond, priv. comm.). This represents a good approximation for shock velocities $\lesssim 200 \mathrm{~km} \mathrm{~s}^{-1}$ since dust is the main coolant only for faster shock waves (Draine 1981), and the dust feedback into the shock structure is negligible.

In this study we have updated and extended the original code (now called GRASH_EX). In this section we describe the new physics and features. We model the carbonaceous grains by replacing graphite/a-C with (hydrogenated) amorphous carbon, a$\mathrm{C}(: \mathrm{H})$ and by considering a molecular approach to the small grain destruction. Furthermore, we model the destruction of silicates taking their core-mantle structure into account. We update the grain charge treatment replacing the McKee et al. (1987) analytical charge scheme to the Weingartner \& Draine (2001) approach and treat thermal and inertial sputtering as a single process. Finally we couple the GRASH_EX code with the DustEM $\operatorname{code}^{2}$ in order to predict the post-shock dust emission and extinction in the ISM.

\subsection{Dust composition effects}

Since the formulation of the MRN model, our understanding of the dust composition has made significant progress. Recent dust models have adopted amorphous carbonaceous grains instead of graphitic-type grains and include aromatic-rich nanoparticles such as polycyclc aromatic hydrocarbons (PAHs) or arophatic clusters (Micelotta et al. 2012). Furthermore the Jones et al. (2013) model, in order to take into account dust evolution in the ISM, considered a-C coated silicate grains. It is therefore necessary to update the modelling of dust destruction in order to properly account for the proposed core/mantle dust composition.

\subsection{1. (Hydrogenated) amorphous carbon sputtering}

In interstellar shocks the gas temperature can be as high as $\sim 5 \times 10^{5} \mathrm{~K}$ and grains therefore undergo sputtering due to collisions with the hot ionised gas. The sputtering of graphitictype grains can be estimated following Tielens et al. (1994). In this study they adopted an analytical function for the sputtering yield which depends on the grain physical parameters and fits the experimental data well with only one free parameter, $K$ (Tielens et al. 1994, Eq. (4.13)). This model was then updated by Serra Díaz-Cano \& Jones (2008), based on the ideas of Jurac et al. (1998), in order to calculate the size-dependent sputtering yield of a-C(:H) grains. In Table 2 we summarise the main parameters for the two materials based on studies by Tielens et al. (1994) for graphitic-type grains and by Serra Díaz-Cano \& Jones (2008) for a-C(:H) grains. $\rho$ is the density of the material, $X_{\mathrm{H}}$ is the $\mathrm{H}$ atom fraction, $U_{0}$ the surface binding energy, $\mathrm{K}$ is a free parameter in this formalism and $\left\langle Z_{2}\right\rangle$ and $\left\langle M_{2}\right\rangle$ are the average

\footnotetext{
2 The DustEM code (Compiègne et al. 2011, http://www.ias. $\mathrm{u}$-psud.fr/DUSTEM) is a FORTRAN code that calculates the dust emission and extinction for a given dust size distribution and interstellar radiation field.
}

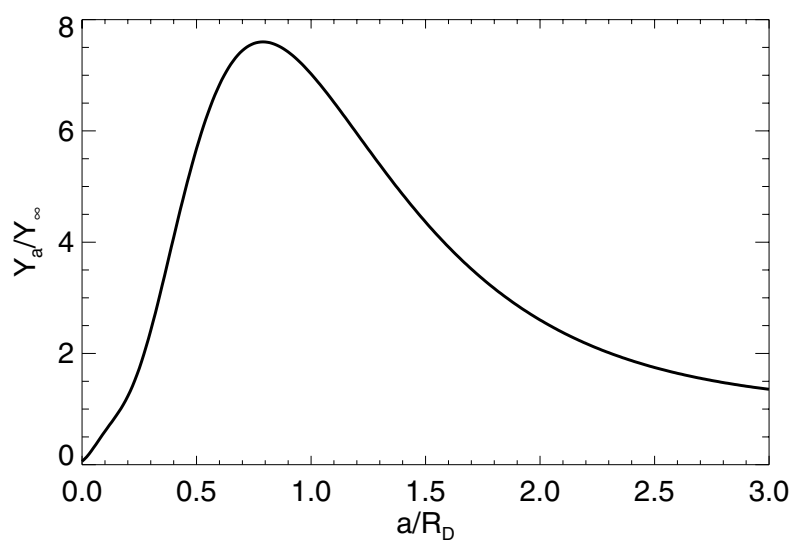

Fig. 2. Sputtering yield ratio $Y_{\mathrm{a}} / Y_{\infty}$ as a function of $a / R_{\mathrm{D}}$ (Serra Díaz-Cano \& Jones 2008). See text for details.

atomic number and mass of the target atoms ${ }^{3}$. We note in particular that there is a big difference in the density between the two materials. Serra Díaz-Cano \& Jones (2008) showed that, regardless the $\mathrm{H}$ atom fraction of the $\mathrm{a}-\mathrm{C}(: \mathrm{H})$ grains, they are much more susceptible to sputtering than graphitic-type grains due to their lower density. Furthermore, Serra Díaz-Cano \& Jones (2008), took into account the finiteness of the a-C $(: \mathrm{H})$ grains (see Fig. 2). We define $Y_{\infty}$ to be the sputtering yield in the case of the sputtering from a semi-infinite plane, $Y_{\mathrm{a}}$ the sputtering of a grain of radius $a$ and $R_{\mathrm{D}}=0.7 R_{\mathrm{p}}$ with $R_{\mathrm{p}}$ the implanted ion penetration depth. Serra Díaz-Cano \& Jones (2008) showed that for small grains, with respect to the penetration depth $\left(a / R_{\mathrm{D}} \ll 0.2\right)$, the projectiles pass through the grain causing little sputtering. On the other hand, for a grain of size similar to the implantation depth, they noted an enhancement of the sputtering yield by a factor of almost 8 with respect to the case of sputtering of a semi-infinite plane, which is due to sputtering from the entire grain surface. However, the sputtering yield for sufficiently large grains is comparable to the sputtering yield in the case of a semi-infinite plane $\left(Y_{\mathrm{a}} / Y_{\infty} \sim 1\right)$.

Figures 3 and 4 illustrate the thermal sputtering rate constant per carbon atom, $\frac{1}{N_{\mathrm{C}} n_{\mathrm{H}}} \frac{\mathrm{d} N_{\mathrm{sp}}}{\mathrm{d} t}$, for grains of radius $a=13 \mathrm{~nm}$ and $a=130 \mathrm{~nm}$, respectively, for different projectile atoms/ions $(\mathrm{H}, \mathrm{He}, \mathrm{C}, \mathrm{N}$ and $\mathrm{O})$ as a function of the gas temperature, $T_{\mathrm{gas}}$. The blue solid lines refer to the estimate by Tielens et al. (1994) for a graphitic-type grain, the black lines refer to the erosion rate of a-C:H grains without any correction due to the size while red lines refer to a-C:H grains with the size effect included. We notice that, due to the lower density of a-C:H with respect to graphite, without including the correction due to the finiteness of the grain, the rate constant for a-C:H grains is about an order of magnitude larger than the rate constant for graphitic grains in the range of temperatures $T=10^{4}-10^{8} \mathrm{~K}$.

Furthermore, comparing the thermal sputtering with and without the size effect included (red and black lines) for both $a=13$ and $130 \mathrm{~nm}$ (see Figs. 3 and 4), we note that for low temperatures $\left(T_{\text {gas }} \sim 10^{4}-10^{5} \mathrm{~K}\right)$, the inclusion of the size effect does not affect the rate constant. On the other hand, for intermediate temperatures $\left(T_{\text {gas }} \sim 10^{6}-5 \times 10^{7} \mathrm{~K}\right)$, a significant enhancement of the rate constant is seen, which corresponds to the enhancement in the sputtering yield due to the finiteness of the grain. Then, for high temperatures $\left(T_{\text {gas }}>2 \times 10^{7} \mathrm{~K}\right.$ for

\footnotetext{
3 The average atomic number and mass of the target atoms are related to the $\mathrm{H}$ atom fraction $\left(X_{\mathrm{H}}=\frac{N_{\mathrm{H}}}{N_{\mathrm{H}}+N_{\mathrm{C}}}\right)$ by: $\left\langle Z_{2}\right\rangle=\left[X_{\mathrm{H}} Z_{\mathrm{H}}+\left(1-X_{\mathrm{H}}\right) Z_{\mathrm{C}}\right]$, $\left\langle M_{2}\right\rangle=\left[X_{\mathrm{H}} M_{\mathrm{H}}+\left(1-X_{\mathrm{H}}\right) M_{\mathrm{C}}\right]$.
} 


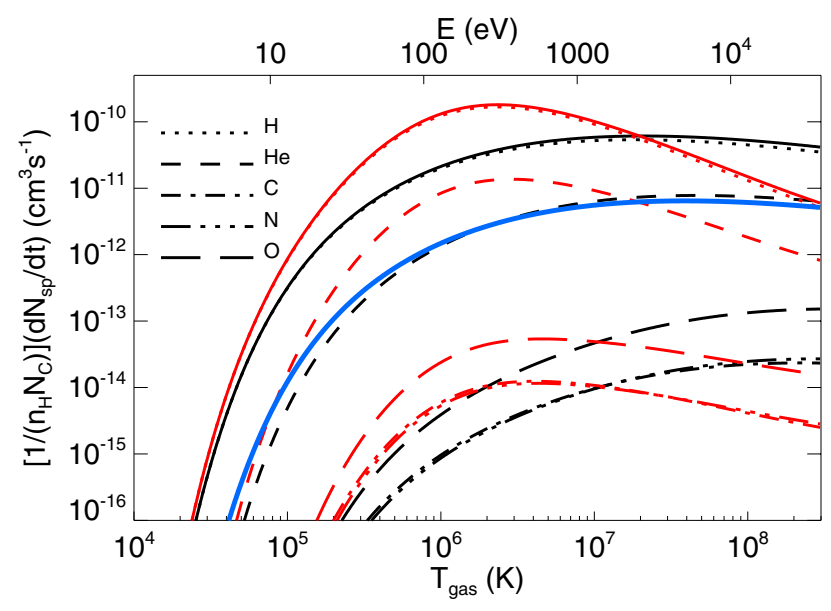

Fig. 3. Thermal sputtering rate constant for an a-C:H grain of radius $13 \mathrm{~nm}$ with (red lines) and without (black lines) the size correction included for different projectiles (the total sputtering is indicated by solid lines). The blue solid line refers to the erosion rate for a graphitic-type grain of radius $13 \mathrm{~nm}$.

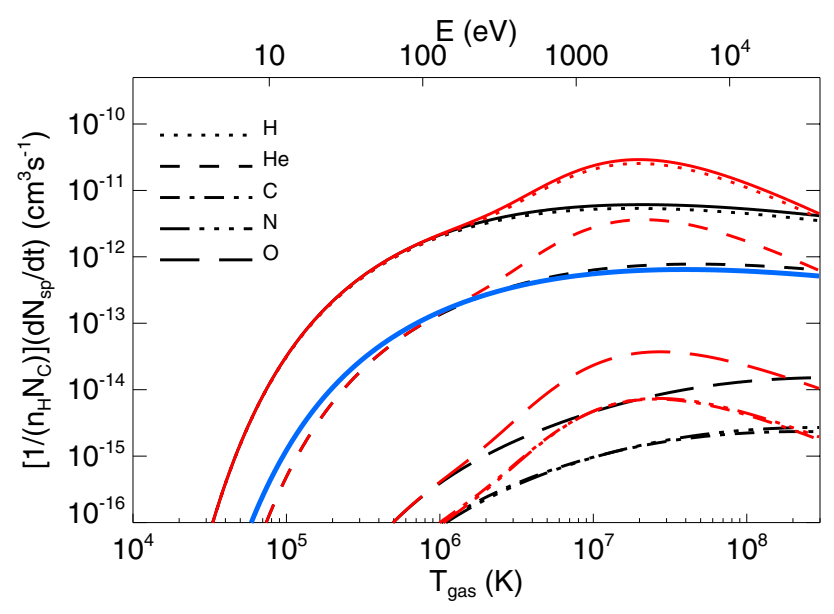

Fig. 4. Same as per Fig. 3 but for a grain of radius $130 \mathrm{~nm}$.

$13 \mathrm{~nm}$ and $T_{\text {gas }}>3 \times 10^{8} \mathrm{~K}$ for $130 \mathrm{~nm}$ ), the penetration depth is larger than the grain size and the inclusion of the size effect in the calculation of the sputtering rate leads to a reduction in the rate constant.

\subsubsection{Amorphous carbon fragmentation and vapourisation}

Following the collision between grains, depending on the energy of the impact, the grains can undergo fragmentation or even vapourisation. Serra Díaz-Cano \& Jones (2008) deduced the parameters for fragmentation and vapourisation for a-C:H grains scaling the parameters used by Tielens et al. (1994) according to the physical properties of a-C:H and graphitic grains. The parameters that we use for a-C:H and graphitic grains are summarised in Table 3. For a detailed explanation of the deduced parameters see Appendix B. We note that fragmentation and vapourisation are more effective at lower energies for a-C $(: \mathrm{H})$ grains than for graphitic grains, which leads to stronger effect in shock waves.

The parameters for fragmentation and vapourisation due to collisions between grains are based on the physics of collisions between hard spheres. However, the small carbonaceous grains we consider in the Jones et al. (2013) dust model are in the
Table 3. Parameters for vapourisation and shattering of graphitic-type and $\mathrm{a}-\mathrm{C}: \mathrm{H}$ grains.

\begin{tabular}{ccccc}
\hline \hline & \multicolumn{2}{c}{ Fragmentation } & \multicolumn{2}{c}{ Vapourisation } \\
Parameter & Graphite & a-C:H & Graphite & a-C:H \\
\hline$\rho_{0}\left[\mathrm{~g} \mathrm{~cm}^{-3}\right]$ & 2.2 & 1.4 & 2.2 & 1.4 \\
$c_{0}\left[\mathrm{~km} \mathrm{~s}^{-2}\right]$ & 1.8 & 1.0 & 1.8 & 1.0 \\
$s$ & 1.9 & 1.9 & 1.9 & 1.9 \\
$P_{\text {th }}\left[\right.$ dyn cm $\left.^{-2}\right]$ & $0.4 \times 10^{11}$ & $0.8 \times 10^{11}$ & $5.8 \times 10^{12}$ & $2.8 \times 10^{12}$ \\
$\epsilon_{\mathrm{v}}\left[\mathrm{erg} \mathrm{g}^{-1}\right]$ & $1.8 \times 10^{9}$ & $0.75 \times 10^{9}$ & $6.4 \times 10^{11}$ & $5.2 \times 10^{11}$ \\
$v_{\text {th }}\left[\mathrm{km} \mathrm{s}^{-1}\right]$ & 1.2 & 0.8 & 23 & 20 \\
$E_{\mathrm{b}}[\mathrm{eV}]$ & 0.02 & 0.01 & 8 & 6.5 \\
\hline
\end{tabular}

molecular regime and cannot be considered as hard spheres. Therefore this approach is probably not valid in the case of collisions between nano-particles and large grains (see discussion in Sect. 5).

\subsubsection{Small carbonaceous grain destruction}

The erosion of polycyclic aromatic hydrocarbons (PAHs), nmsize particles, in a hot gas has been studied in detail by Micelotta et al. (2010b). They considered dissociative sputtering resulting from the combination of nuclear interaction and electronic interactions, and electron collisions. They showed that the destruction of very small carbonaceous grains, for temperatures $T_{\text {gas }} \leqslant 5 \times 10^{7} \mathrm{~K}$, is dominated by electron collisions. On the other hand, the sputtering of larger grains $(a \gtrsim 3 \mathrm{~nm}$, corresponding to $N_{\mathrm{C}} \gtrsim 900$ ) is dominated by nuclear and electronic interactions.

Bocchio et al. (2012) showed that, the sputtering rate constant for $T_{\text {gas }} \lesssim 10^{7} \mathrm{~K}$ and $a \gtrsim 3 \mathrm{~nm}$, calculated using the molecular approach of Micelotta et al. (2010) and the classical approach used by Tielens et al. (1994) and Serra Díaz-Cano \& Jones (2008) are the same to within a factor two. Therefore, in the GRASH_EX code, the large grain sputtering is treated using the classical approach while the sputtering of grains with $a \lesssim 3 \mathrm{~nm}$ is treated using the molecular approach.

\subsubsection{Treatment of the core-mantle structure of grains}

As presented in Sect. 2, in the Jones et al. (2013) dust model both carbonaceous and silicate grains have core-mantle structure and we need to use two different approaches for the two materials.

The sputtering yield of amorphous carbon is little affected by its $\mathrm{H}$ atom content (Serra Díaz-Cano \& Jones 2008) thus the aliphatic-rich core and the aromatic-rich mantle of the carbonaceous grains are treated in the same way. We therefore do not keep track of the thickness of the aromatic mantle on the carbonaceous grains.

The treatment of silicate grains must take account of the different mantle and core sputtering properties; silicate core with a carbonaceous mantle. However, to keep track of the mantle thickness on the surface of the silicate grain core throughout the whole shock, and for each bin in the mass distribution, is difficult to keep track of and is time consuming. We therefore make an approximation: during the time needed to destroy the a-C mantle, the grain core is little affected and none of its atoms is ejected. To check whether this assumption is valid, we use 
Table 4. SRIM calculations (based on $10^{5}$ collisions) of the silicate core sputtering yield, $Y_{\mathrm{i}}$, due to bombardment by $\mathrm{H}^{+}$and $\mathrm{He}^{+}$ions.

\begin{tabular}{c|cc}
\hline \hline & \multicolumn{2}{|c}{$Y_{\mathrm{i}}$} \\
Mantle thickness & $\mathrm{H}^{+}$ & $\mathrm{He}^{+}$ \\
\hline $5 \mathrm{~nm}$ & $<10^{-5}$ & $<10^{-5}$ \\
$1 \mathrm{~nm}$ & $<10^{-5}$ & $\sim 4.310^{-4}$ \\
$0 \mathrm{~nm}$ & $<10^{-5}$ & $\sim 5.510^{-2}$ \\
\hline
\end{tabular}

the Stopping and Range of Ions in Matter software ${ }^{4}$ (SRIM, Ziegler et al. 1985) to simulate the sputtering of core-mantle silicate grains due to collisions with $\mathrm{H}^{+}$and $\mathrm{He}^{+}$ions (i.e. the ions that dominate the sputtering process). We consider a thick semiinfinite plane made of amorphous forsterite-type silicate covered by a thin layer of amorphous carbon. We vary the carbonaceous mantle thickness in order to mimic the destruction of the mantle. The relevant parameters involved in the sputtering processes are: the density of the material, $\rho$, the surface binding energy, $E_{\mathrm{S}}$, the displacement energy, $E_{\mathrm{D}}$, and the lattice binding energy, $E_{\mathrm{B}}$ (see Sect. 5.1 for details). Despite the uncertainties on these parameters (see Sect. 5.1) we are here interested in the relative sputtering yield for different mantle thicknesses and not in their absolute values. We use here the parameters adopted by Serra Diaz-Cano \& Jones 2008 for amorphous carbon and the parameters presented in Table 8 for silicate grains. We consider a maximum shock velocity of $200 \mathrm{~km} \mathrm{~s}^{-1}$. In this case the energy of the impinging $\mathrm{H}^{+}$and $\mathrm{He}^{+}$ions reaches at most $\sim 100 \mathrm{eV}$ and $\sim 470 \mathrm{eV}$ respectively. We use these energies for all the simulations presented in this section.

We simulate $10^{5}$ collisions and average the resulting sputtering yield depending on the atomic mass of the sputtered atom from the silicate core (see Sect. 5.1, Eq. (23)). In Table 4 we report the obtained silicate sputtering yield for an a-C mantle thickness varying from $5 \mathrm{~nm}$ to $0 \mathrm{~nm}$ in order to mimic the destruction of the mantle. We notice that the sputtering yield for $\mathrm{H}^{+}$projectiles is negligible even for bare silicate grains, while the sputtering yield for $\mathrm{He}^{+}$is highly affected by the presence of the mantle; even in the case of $1 \mathrm{~nm}$ carbonaceous mantle, the sputtering yield is two orders of magnitude lower than in the case of bare silicates. This leads us to the conclusion that our approximation is valid.

We then define a time needed for the carbonaceous mantle to be completely destroyed, $t_{\text {man }}$. We run the GRASH_EX code a first time for different shock velocities to estimate $t_{\text {man }}$ for a silicate grain of radius $a=160 \mathrm{~nm}$, i.e. the most abundant silicate grains in the Jones et al. (2013) dust model. We then consider that the silicate grains are not eroded until a time $t_{\text {man }}$ has passed (in order to mimic the effect of carbonaceous mantles on silicate grains) and then remove the mantles and allow silicate grain sputtering. In Table 5 we summarise $t_{\text {man }}$ for a grain of radius $a=160 \mathrm{~nm}$ for different shock velocities. It is clear that $t_{\text {man }}$ for all of the shock velocities is much shorter than the typical shock timescale (i.e. $\sim 10^{5} \mathrm{yr}$ ). Therefore the presence of a carbonaceous mantle is not able to significantly protect silicate grains from erosion.

\footnotetext{
4 SRIM is a collection of software packages which calculates the transport of ions in matter, calibrating the results on the available experimental data.
}

Table 5. $t_{\operatorname{man}}(\mathrm{yr})$ for different shock velocities $\left(\mathrm{km} \mathrm{s}^{-1}\right)$.

\begin{tabular}{c|cccc}
\hline \hline$V_{\text {shock }}$ & 50 & 75 & 100 & 125 \\
$t_{\text {man }}$ & $2.0 \times 10^{4}$ & $1.8 \times 10^{3}$ & $1.0 \times 10^{3}$ & $7.1 \times 10^{2}$ \\
\hline \multicolumn{5}{c}{} \\
\hline$V_{\text {shock }}$ & 150 & 175 & 200 \\
$t_{\text {man }}$ & $1.1 \times 10^{3}$ & $8.8 \times 10^{2}$ & $4.0 \times 10^{2}$ & \\
\hline
\end{tabular}

\subsection{Grain charge}

The acceleration, $\mathrm{d} V_{\text {rel }} / \mathrm{d} t$, of a grain with respect to a shocked gas can be expressed as

$$
\frac{\mathrm{d} V_{\text {rel }}}{\mathrm{d} t}=\frac{\mathrm{d} \chi}{\mathrm{d} t} \frac{V_{\text {rel }}}{2 \chi}-\frac{F_{\mathrm{D}}}{m}
$$

The first term on the right (Cowie 1978; Shull 1978) is called betatron acceleration and is proportional to the time-derivative of the magnetic field strength (related to the shock compression $\chi$ ). This acceleration is due to the gyration velocity around a magnetic field line. The second term is the drag deceleration, where $F_{\mathrm{D}}$ is the drag force and $m$ the mass of the grain. The drag force was evaluated by Draine \& Salpeter (1979a) as

$F_{\mathrm{D}}=F_{\mathrm{D}}($ direct $)+F_{\mathrm{D}}($ plasma $)$

where $F_{\mathrm{D}}$ (direct) is the direct collisional drag and $F_{\mathrm{D}}$ (plasma) is the plasma drag. While the direct collisional drag only depends on the geometrical cross section and mass of projectiles and target, the plasma drag, which strongly depends on the grain charge, can be expressed as

$F_{\mathrm{D}}($ plasma $)=4 \pi a^{2} k_{\mathrm{B}} T_{\text {gas }} \phi^{2} \ln \Lambda \sum_{i} n_{i} z_{i}^{2} H\left(s_{i}\right)$,

where $a$ is the grain radius, $k_{\mathrm{B}}$ is the Boltzmann constant, $T_{\text {gas }}$ the gas temperature, $\ln \Lambda$ the Coulomb logarithm, $n_{i}$ and $z_{i}$ the density and charge of the $i$ th ion. The electric potential parameter $\phi$ is expressed as

$\phi \equiv \frac{z_{\mathrm{g}} e^{2}}{a k_{\mathrm{B}} T_{\mathrm{gas}}}$,

with $z_{\mathrm{g}}$ the grain charge and the function $H\left(s_{i}\right)$ given by

$H\left(s_{i}\right)=s_{i}\left(\frac{3}{2} \sqrt{\pi}+2 s_{i}^{3}\right)^{-1}$,

where $s_{i}$ is defined as

$s_{i}^{2}=\frac{m_{i} V_{\mathrm{rel}}^{2}}{2 k_{\mathrm{B}} T_{\mathrm{gas}}}$,

with $m_{i}$ the mass of the $i$ th ion.

In the original work by Jones et al. $(1994,1996)$ the grain charge was calculated using the analytical charge scheme of McKee et al. (1987), which was based on calculations by Draine (1981) and Draine \& Salpeter (1979b). A more recent study by (Weingartner \& Draine 2001, WD01) uses a better estimate of the electron sticking coefficient and the photoelectric yield and is based on experimental results.

We find that this update to the grain charge calculation leads to only small variations in the grain potential and therefore little affects the dust dynamics. Figure 5 shows the potential parameter $\phi$ (see Eq. (4)) as a function of the gas temperature, $T_{\text {gas }}$ for 


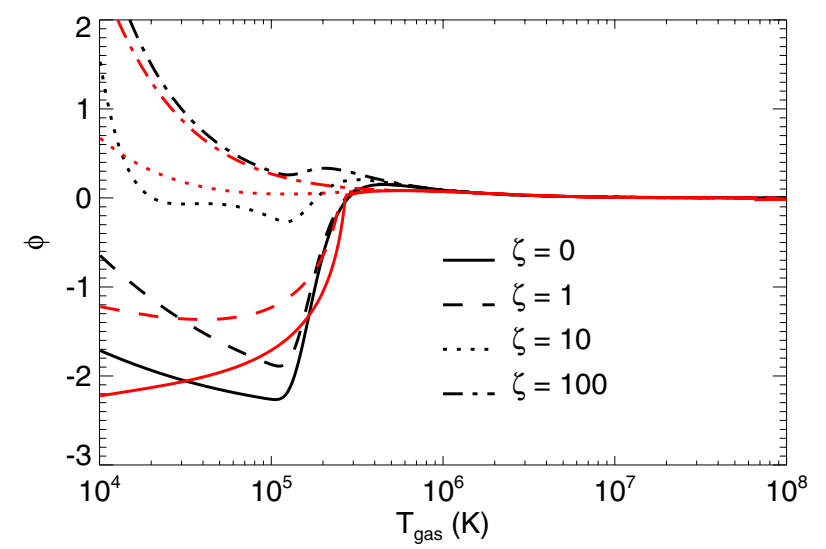

Fig. 5. Potential parameter $\phi=\frac{z_{\mathrm{g}} e^{2}}{a k_{\mathrm{B}} T_{\mathrm{gas}}}$ as a function of the gas temperature for a $160 \mathrm{~nm}$ silicate grain. Different values of $\zeta$ (see text for details) are labeled with different line styles and the black and red curves refer to the McKee et al. (1987) analytical scheme and to the WD01 charge scheme, respectively.

a silicate grain of radius of $160 \mathrm{~nm}$. The potential is calculated using both the analytical scheme of McKee et al. (1987) (black lines) and the more recent estimates by WD01 (red lines). The $\zeta$ parameter was introduced by McKee et al. (1987) and is defined as

$\zeta=\frac{G_{0}}{n_{\mathrm{e}}}\left(\frac{a}{10 \mathrm{~nm}+a}\right)$,

where $G_{0}$ is the incident UV intensity normalised to the average interstellar background flux $\left(2.4 \times 10^{6} \mathrm{~cm}^{-2} \mathrm{~s}^{-1}\right)$ adopted by Draine \& Salpeter (1979b) and $n_{\mathrm{e}}$ is the electron density in the gas. In Fig. 5 we consider $\zeta=0,1,10$ and 100 and assume that the gas is fully ionised $\left(n_{\mathrm{e}}=n_{\mathrm{H}}\right)$. In the GRASH_EX code we have updated the grain charge calculation to the WD01 approach.

\subsection{The skewed Maxwellian distribution}

In the formalism introduced by Tielens et al. (1994) a distinction is made between thermal sputtering (due to a hot gas) and the inertial sputtering of a grain as a function of its drift velocity in the gas. The thermal sputtering rate can be expressed as

$\frac{\mathrm{d} N_{\mathrm{sp}}}{\mathrm{d} t}=2 \pi a^{2} \sum_{i} n_{i}\left\langle Y_{i} v\right\rangle$,

where $\frac{\mathrm{d} N_{\mathrm{sp}}}{\mathrm{d} t}$ is the number of sputtered atoms per unit time, $a$ is the grain radius, $n_{i}$ the density of the $i$ th ion in the gas and

$\left\langle Y_{i} v\right\rangle=\int Y_{i} v f_{\mathrm{M}}(v) \mathrm{d} v$,

where $Y_{i}$ is the sputtering yield of the $i$ th ion, $v$ is the relative (thermal) velocity between the $i$ th ion and the target grain and $f_{\mathrm{M}}(v)$ is the Maxwellian distribution for a velocity $v$.

The non-thermal or inertial sputtering rate can be expressed as

$\frac{\mathrm{d} N_{\mathrm{sp}}}{\mathrm{d} t}=2 \pi a^{2} v_{\mathrm{g}} \sum n_{i} Y_{i}\left(E=0.5 m_{i} v_{\mathrm{g}}^{2}\right)$.

This corresponds to a thermal sputtering rate where the function used to average the sputtering yield is not the Maxwellian distribution but a Dirac delta function centred at the drift velocity of the grain in the gas, $v_{\mathrm{g}}$.

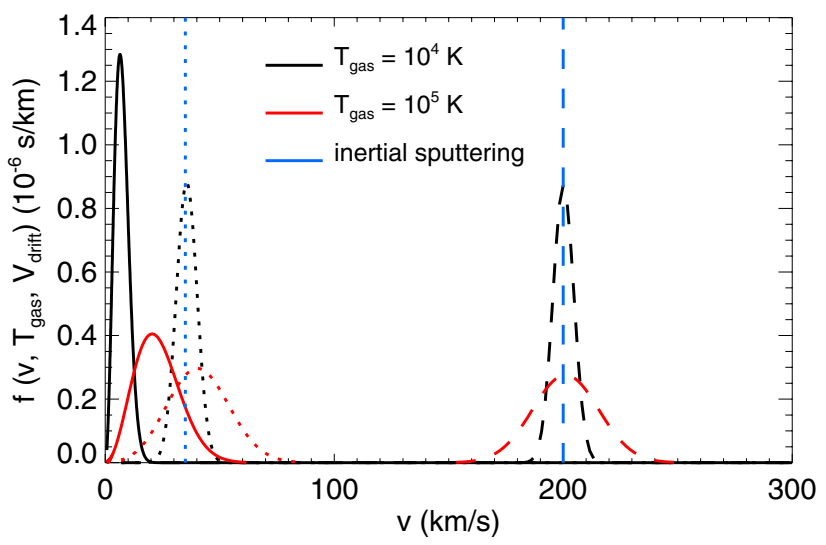

Fig. 6. Skewed Maxwellian distribution as a function of the relative velocity between a grain and the gas for different drift velocities and gas temperatures. Solid lines show the case of $V_{\text {drift }}=0 \mathrm{~km} \mathrm{~s}^{-1}$, dotted lines the case of $V_{\text {drift }}=35 \mathrm{~km} \mathrm{~s}^{-1}$ and dashed lines the case of $V_{\text {drift }}=200 \mathrm{~km} \mathrm{~s}^{-1}$. Black lines are for a gas temperature $T_{\text {gas }}=10^{4} \mathrm{~K}$, red lines are for $T_{\mathrm{gas}}=10^{5} \mathrm{~K}$ and the blue lines represent the inertial sputtering.

However, inertial sputtering is only an approximation and in reality, the gas is at a given temperature and the relative velocity between a grain and the surrounding ions is not unimodal but it is a combination of the thermal motion of the ions and the drift of the grain in the gas. This motion is well described by the skewed Maxwellian distribution (Shull 1978; Guillet 2008, see Appendix A for details).

$$
\begin{aligned}
f_{\text {skM }}= & \sqrt{\frac{m_{\mathrm{i}}}{2 \pi k_{\mathrm{B}} T_{\text {gas }}}} \frac{v}{V_{\text {drift }}}\left[\exp \left(-\frac{m_{\mathrm{i}}}{2 k_{\mathrm{B}} T_{\text {gas }}}\left(v-V_{\text {drift }}\right)^{2}\right)\right. \\
& \left.-\exp \left(-\frac{m_{\mathrm{i}}}{2 k_{\mathrm{B}} T_{\text {gas }}}\left(v+V_{\text {drift }}\right)^{2}\right)\right]
\end{aligned}
$$

where $f_{\mathrm{skM}}$ is the velocity probability function, $m_{\mathrm{i}}$ is the $i$ th ion mass, $k_{\mathrm{B}}$ is the Boltzmann constant, $T_{\text {gas }}$ is the gas temperature, $v$ is the relative velocity between the ions and the grain and $V_{\text {drift }}$ is the drift velocity of the grain in the gas. Replacing $f_{\mathrm{M}}$ with $f_{\mathrm{skM}}$ in Eq. (9) and using this expression in Eq. (8) we obtain the erosion rate of a grain in the case of a grain drifting in a gas whose ions are in random thermal motion at a given temperature $T_{\text {gas }}$.

Figure 6 shows the skewed Maxwellian distribution for $V_{\text {drift }}=0,35$ and $200 \mathrm{~km} \mathrm{~s}^{-1}$ for temperatures $T_{\text {gas }}=10^{4} \mathrm{~K}$ and $10^{5} \mathrm{~K}$ as a function of the relative velocity between a grain and an ion in the gas. We compare them to the corresponding Dirac delta functions at $V_{\text {drift }}=35$ and $200 \mathrm{~km} \mathrm{~s}^{-1}$. We notice that the Dirac delta function is a good approximation for $T_{\text {gas }}=10^{4} \mathrm{~K}$ but for $T_{\text {gas }}=10^{5} \mathrm{~K}$ the broadening of the distribution function and the shift of the peak position become important.

In the original code (Jones et al. 1994, 1996) thermal and inertial sputtering were assumed to be de-coupled. The thermal sputtering was neglected for a gas temperature $T_{\text {gas }}<10^{4} \mathrm{~K}$, while the inertial sputtering was considered only in the case of a drift velocity $V_{\text {drift }}>0.1 \mathrm{~km} \mathrm{~s}^{-1}$. In the GRASH_EX code these two processes are coupled using the skewed Maxwellian distribution described above.

To show the effect of the skewed Maxwellian distribution with respect to the Dirac delta function we consider the astrophysical relevant case of sputtering of a graphitic grain due to bombardment of $\mathrm{H}, \mathrm{He}$ and $\mathrm{C}$ projectiles. As assumed by Bocchio et al. (2012), we consider the relative abundances 1, 0.1 and $10^{-4}$ for $\mathrm{H}, \mathrm{He}$ and $\mathrm{C}$, respectively. In Fig. 7 we plot the 
Marco Bocchio et al.: A re-evaluation of dust processing in supernova shock waves

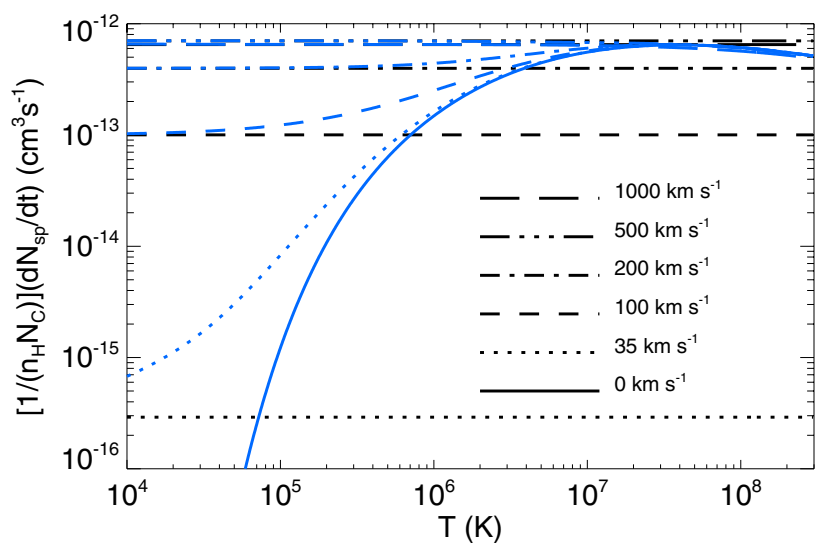

Fig. 7. $\frac{1}{N_{\mathrm{C}} n_{\mathrm{H}}} \frac{\mathrm{d} N_{\mathrm{Sp}}}{\mathrm{d} t}$ as a function of the gas temperature for a graphitictype grain of radius $130 \mathrm{~nm}$ for different relative velocities between the projectiles $(\mathrm{H}, \mathrm{He}$ and $\mathrm{C})$ and the target grain. Blue lines show the erosion rate computed using the skewed Maxwellian distribution while black lines show the inertial sputtering formulation.

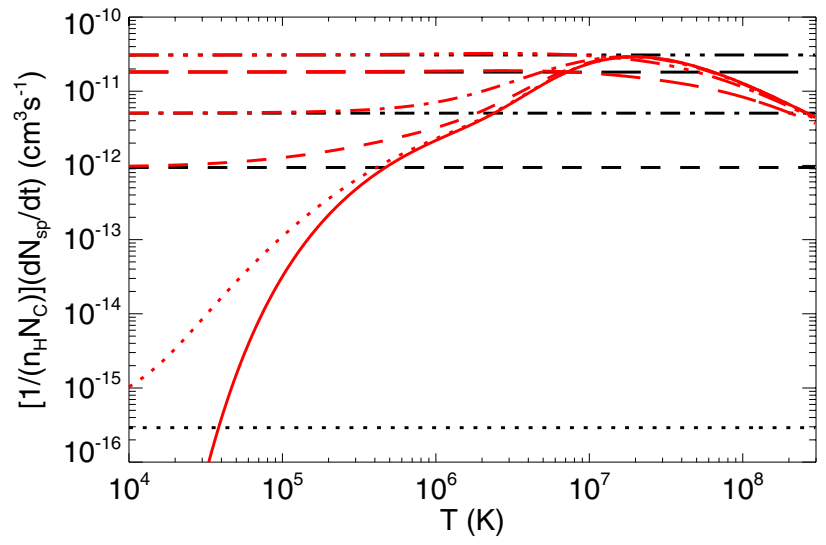

Fig. 8. Erosion rate for an a-C:H grain of radius $130 \mathrm{~nm}$ for different drift velocities between the projectiles and the target grain. Red lines represent the erosion rate computed using the skewed Maxwellian distribution while black lines with the inertial sputtering formulation. The line style coding is the same as for Fig. 7.

sputtering rate constant per carbon atom for a graphitic grain of radius $a=130 \mathrm{~nm}$ as a function of the gas temperature for different $V_{\text {drift }}$. We calculate the sputtering rate using the classical inertial sputtering (i.e. using a Dirac delta distributed velocity) and using the skewed Maxwellian distribution. We clearly see in Fig. 7 that the inertial sputtering is not a good approximation at low drift velocities ( $V_{\text {drift }} \lesssim 100 \mathrm{~km} \mathrm{~s}^{-1}$ ) while it is a good approximation at higher velocities. In particular, for a drift velocity of $35 \mathrm{~km} \mathrm{~s}^{-1}$ and for $T \lesssim 10^{5} \mathrm{~K}$, the sum of the thermal sputtering (blue solid line) and inertial sputtering (black dotted line) is lower, by a factor of 2 to 10 , than the sputtering calculated using the skewed Maxwellian distribution (blue dotted line). This difference is due to the relevant high-energy tail in the case of the skewed Maxwellian approach (see Fig. 6).

Figure 8 shows the sputtering rate constant per carbon atom for an a-C:H grain of radius $a=130 \mathrm{~nm}$ as a function of the gas temperature with the inclusion of the size effect, which leads, as noticed in Sect. 3.1.1, to a lowering of the sputtering yield for high gas temperatures $\left(T_{\text {gas }} \gtrsim 10^{7} \mathrm{~K}\right)$. In this case, the inertial sputtering, even for the highest velocities considered $\left(V_{\text {rel }}=1000 \mathrm{~km} \mathrm{~s}^{-1}\right)$, is not a good approximation and the skewed Maxwellian distribution should be used instead.

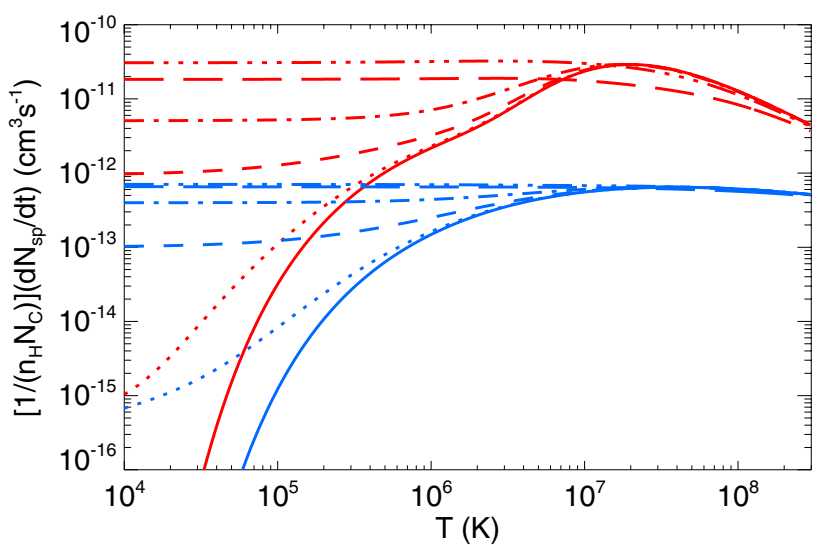

Fig. 9. Erosion rate for an a-C:H (red lines) and a graphitic-type (blue lines) grain of radius $130 \mathrm{~nm}$ for different drift velocities between the projectiles and the target grain. The line style coding is the same as for Figs. 7 and 8.

Finally, to directly compare the erosion of a graphitic grain and an a-C:H grain in Fig. 9 we illustrate the sputtering rate constant for both grains and for different $V_{\text {drift }}$ as a function of the gas temperature. We clearly see that the change in the physical properties of the grains (a-C:H with respect to graphitic-type) together with their finiteness leads to a substantial enhancement (by more than one order of magnitude for some temperatures) in the sputtering rate constant.

\subsection{Interfacing GRASH_EX and DustEM}

A coupling of the GRASH_EX and DustEM codes allows an exchange of the pre- and post-shock size distributions between the codes. This feature is introduced in the new version of the code and allows the user to 1) define any pre-shock size distribution, without being limited to power-law distributed grains and 2) to calculate the dust emission and extinction before and after a shock.

\subsubsection{The dust size distribution}

In the GRASH_EX code we exploit the coupling with the DustEM code to allow the user to define the grain size distribution in the same way as per the DustEM code, i.e. with the use of the GRAIN.DAT file. To keep the structure of the GRASH_EX code relatively simple and optimise the computing time we decided here to limit the choice of the dust model to a two-material dust model. The Jones et al. (2013) dust model includes two different size distributions for the carbonaceous grains; the program automatically merges them together into one distribution in order to yield only two dust populations: carbonaceous and silicate grains.

The GRASH_EX code defines a dust population in terms of mass distribution in order to allow for mass conservation, while the DustEM code defines a dust population in terms of its size distribution. Then, in order to make the two programs interface with each other we wrote a routine to translate a mass distribution into size distribution and vice versa.

To define a mass distribution from a user-supplied size distribution, firstly we define a logarithmic grid in mass with the desired number of bins and with the mass range corresponding to radius range in the size distribution. As in the DustEM code, for each bin, we define the quantity $\frac{a^{4} \mathrm{~d} n}{\mathrm{~d} a}$, where $n$ is the number 


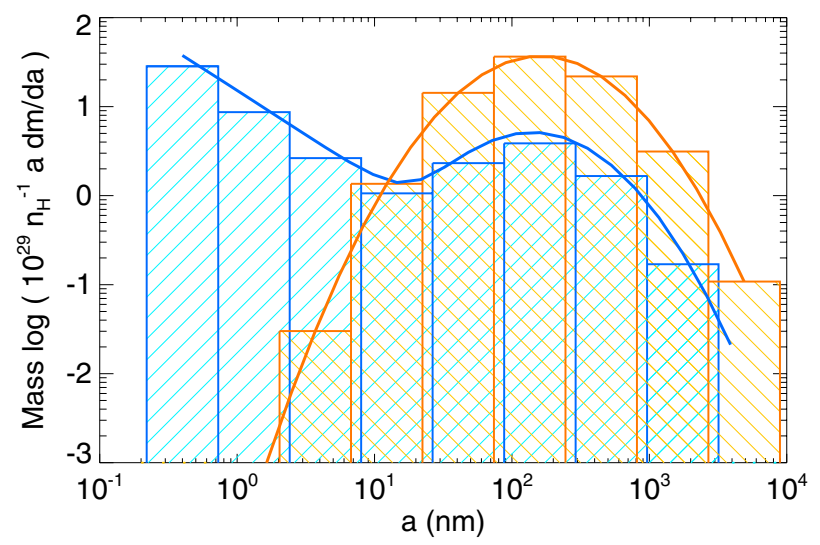

Fig. 10. Jones et al. (2013) dust mass distribution. Blue and orange bins refer to carbonaceous and silicate grains respectively. Here the carbonaceous and silicate grains mass distributions are divided into 9 bins. The solid lines indicate the case of an infinite number of bins.

of grains in the bin and $a$ the grain radius. The total mass, $M_{i}$, in the $i$ th bin can be expressed as

$M_{i}=\int\left(\frac{\mathrm{d} n}{\mathrm{~d} m}\right)_{i} m \mathrm{~d} m$

where $m$ is the grain mass. The fraction $\frac{\mathrm{d} n}{\mathrm{~d} m}$ can be expressed as

$\frac{\mathrm{d} n}{\mathrm{~d} m}=\frac{\mathrm{d} n}{\mathrm{~d} a} \frac{\mathrm{d} a}{\mathrm{~d} m}$,

and since $m=\frac{4}{3} \pi a^{3} \rho$ (where $\rho$ is the grain density) we can write

$\frac{\mathrm{d} m}{\mathrm{~d} a}=4 \pi a^{2} \rho$

and after some algebra we obtain

$\frac{\mathrm{d} n}{\mathrm{~d} m}=\left(\frac{a^{4} \mathrm{~d} n}{\mathrm{~d} a}\right) \frac{4 \pi \rho}{9 m^{2}}$,

and finally

$M_{i}=\int\left(\frac{a^{4} \mathrm{~d} n}{\mathrm{~d} a}\right)_{i} \frac{4 \pi \rho}{9 m} \mathrm{~d} m$.

Figures 10-12 show the mass distribution as a function of the grain mass divided into 9, 15 and 25 bins, respectively. As expected, increasing the number of bins, leads to a mass distribution that is defined with more and more precision, but the computing time to run the code through the whole shock wave increases at least as the square of the number of bins. For our standard run we choose to optimally divide the size distribution into 9 bins. We discuss the effect of the binning interval in Sect. 5.4.

\subsubsection{Dust emission and extinction in shocked regions}

The processing of dust in a shock wave will determine its size distribution in shocked regions in galaxies. Coupling the GRASH_EX code with the DustEM code we have the possibility to predict the dust spectral energy distribution (SED) and extinction from regions that have been shocked, in order to allow comparison with observations. After the dust processing by

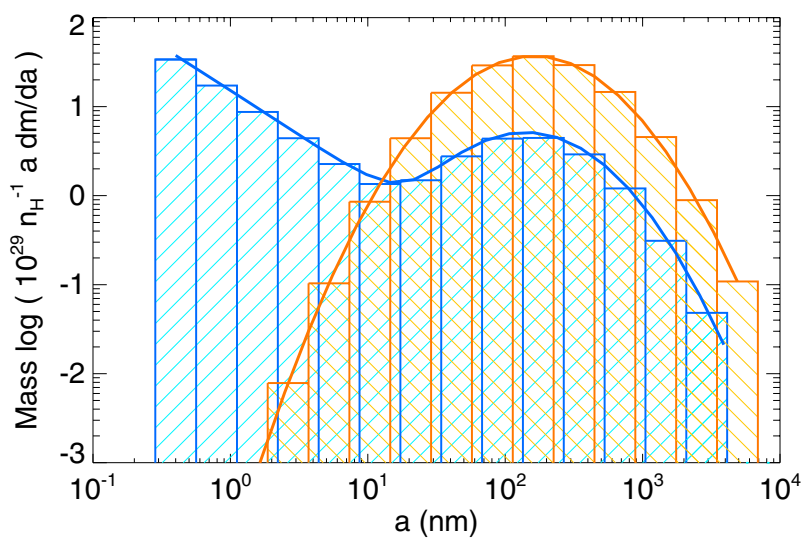

Fig. 11. As per Fig. 10 but for 15 bins.

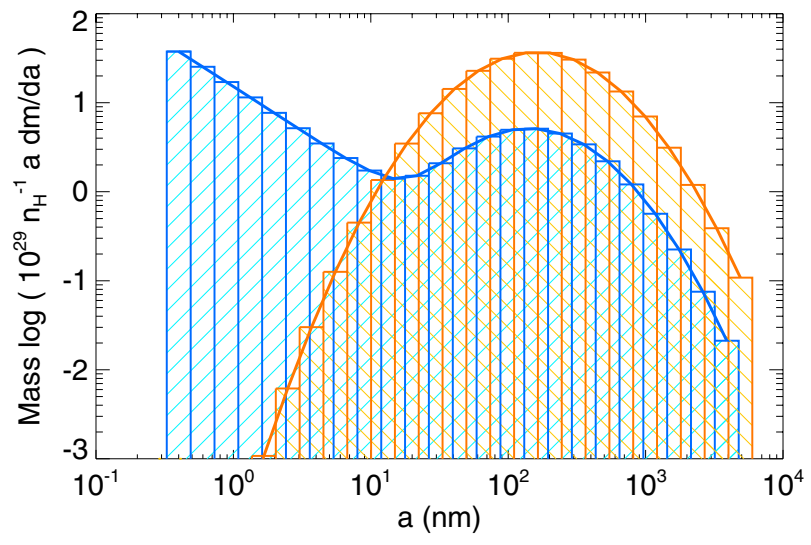

Fig. 12. As per Fig. 10 but for 25 bins.

a given shock wave, the post-shock mass distributions are translated into size distributions that can be read by the DustEM code, as explained in Sect. 3.4.1. In order to generate the dust SED and extinction we need to calculate the dust optical properties. In the Jones et al. (2013) dust model, these are defined by the mantle thickness and the band gap of the carbonaceous material.

\section{Results}

Here we assume shock processing in the WIM (as per McKee et al. 1987; Jones et al. 1994, 1996) and assume pre-shock density, $n_{0}$, and magnetic field, $B_{0}$, values typical of the WIM (i.e. $n_{0}=0.25 \mathrm{~cm}^{-3}$ and $\left.B_{0}=3 \mu \mathrm{G}\right)$. We consider an initial ionisation fraction of $50 \%$, which corresponds to a good approximation, to the precursor ionisation caused by the shock. We choose a shock velocity of $100 \mathrm{~km} \mathrm{~s}^{-1}$ for our standard run since it is close to the optimum of the shock frequency and destructive potential in interstellar shocks (Draine \& Salpeter 1979b,a; Jones et al. 1994, 1996).

We define the column density, $N_{\mathrm{H}}$, through the shock as

$N_{\mathrm{H}}=v_{\text {shock }} n_{0} t_{\text {shock }}$,

with $t_{\text {shock }}$ the time after the beginning of the shock. Figure 13 illustrates the shock profile for our standard shock. We show the gas temperature $\left(T_{4}\right.$, in units of $\left.10^{4} \mathrm{~K}\right)$, density $\left(n_{\mathrm{H}}\right)$ and ionisation fraction $\left(X_{\mathrm{e}}\right)$ along the shock as a function of the column density.

The relative velocity, $V_{\text {rel }}$, between a grain and the gas is a particularly important parameter as it determines the degree of grain destruction. Figure 14 shows the grain drift velocity with 
Marco Bocchio et al.: A re-evaluation of dust processing in supernova shock waves

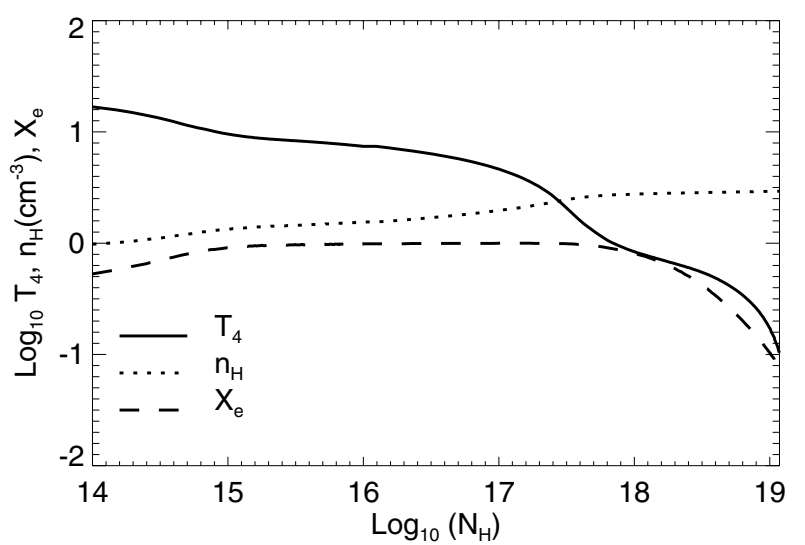

Fig. 13. Shock profile for the standard $100 \mathrm{~km} / \mathrm{s}$ shock. $T_{4} \equiv \frac{T_{\text {gas }}}{10^{4} \mathrm{~K}}, n_{\mathrm{H}}$ and $X_{\mathrm{e}}$ are the temperature (in units of $10^{4} \mathrm{~K}$ ), density and ionisation fraction along the shock.

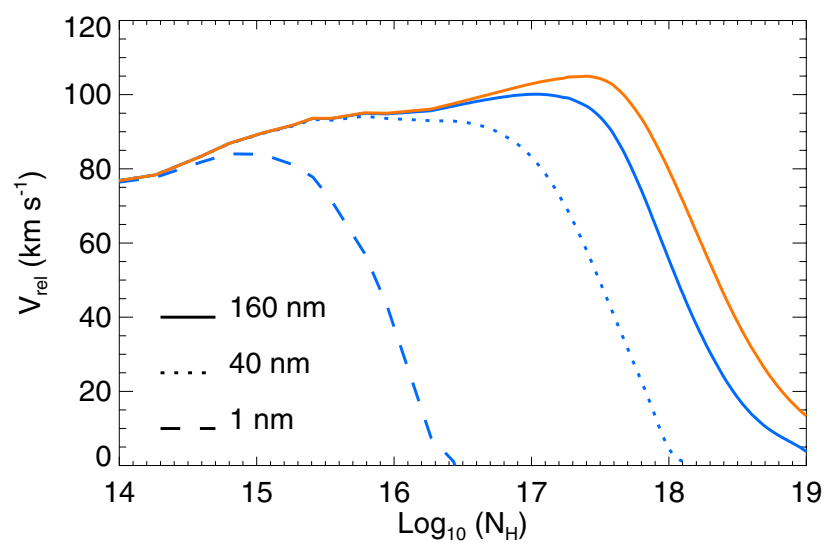

Fig. 14. Relative velocity between a grain and the gas for $v_{\text {shock }}=$ $100 \mathrm{~km} \mathrm{~s}^{-1}$ and for different grain sizes. We adopt the same colourcoding as per Figs. 10-12.

respect to the gas as a function of the column density for our standard shock. We adopt different line styles for different carbonaceous grain sizes as labeled and the same colour-coding as per Figs. 10-12, i.e. blue lines for carbonaceous grains and the orange line for a silicate grain. Focusing on the drift velocity of a $160 \mathrm{~nm}$ grain, we notice that the grain is first accelerated immediately after the shock front $\left(N_{\mathrm{H}} \sim 10^{14}-10^{15} \mathrm{~cm}^{-2}\right)$ because of the sudden change in the gas density (due to ionisation) and later at $N_{\mathrm{H}} \sim 10^{16}-10^{17} \mathrm{~cm}^{-2}$ because of the rapid cooling of the gas and the subsequent shock compression (betatron acceleration, Sect. 3.2). Furthermore, we notice that the higher density of silicate grains lead to a longer coupling time for a $160 \mathrm{~nm}$ silicate grain than for a carbonaceous grain of the same size.

We run the code for different shock velocities: 50, 75, 100, $125,150,175$ and $200 \mathrm{~km} \mathrm{~s}^{-1}$ using the shock profiles generated by (Raymond, priv. comm.). Figure 15 illustrates the post-shock size distribution for $v_{\text {shock }} \leqslant 175 \mathrm{~km} \mathrm{~s}^{-1}$. The post-shock size distribution in the case of a $200 \mathrm{~km} \mathrm{~s}^{-1}$ shock is almost identical to the $175 \mathrm{~km} \mathrm{~s}^{-1}$ case and is not shown in Figs. 15-18. We notice that small carbonaceous grains are highly affected by the shock wave; even for a $50 \mathrm{~km} \mathrm{~s}^{-1}$ shock most of the mass of the smallest grains is transferred to the gas phase. For shock velocities $\geqslant 175 \mathrm{~km} \mathrm{~s}^{-1}$ all the carbonaceous grains are destroyed. On the other hand silicate grains are more resistant to sputtering and therefore undergo less destruction. However, for a $100 \mathrm{~km} \mathrm{~s}^{-1}$ shock, we clearly see a broadening of the silicate grain size distribution due to the fragmentation of the large grains.

\subsection{Dust emission and extinction}

For all the considered shock velocities the carbonaceous mantle on the surface of silicate grains is destroyed (see Sect. 3.1.4). On the other hand, tracking the thickness of the aromatic mantle on the surface of carbonaceous grains is a more difficult task. However, energetic ion collisions and photon irradiation are able to re-aromatise the outer layer of the carbonaceous grains. Firstly, the available timescale is sufficiently long $\left(\sim 10^{5} \mathrm{yr}\right)$ that the radiation field $\left(G_{0}>1\right)$ would aromatise carbonaceous grain surfaces to a maximum depth of $\sim 20 \mathrm{~nm}$ (Jones 2012d; Jones et al. 2014). Furthermore, sufficiently energetic ions can penetrate carbonaceous grains up to $\sim 5 \mathrm{~nm}^{5}$ contributing to the aromatisation of the outer layer. We therefore consider that, for each of the shock velocities, the post-shock silicate grains are bare while carbonaceous grains have a $20 \mathrm{~nm}$ aromatic mantle with an aliphatic rich core for $a>20 \mathrm{~nm}$ and are aromatic-rich for $a<20 \mathrm{~nm}$. We calculate the corresponding optical properties and then, using the post-shock size distribution as an input for the DustEM code, calculate the SED and extinction produced by the dust that has survived. Figure 16 shows the dust SED for $50,75,100,125,150$ and $175 \mathrm{~km} \mathrm{~s}^{-1}$ shocks. The destruction of the smallest (carbonaceous) grains is reflected in the dust SED: even after a $50 \mathrm{~km} \mathrm{~s}^{-1}$ shock we clearly see a drop in the emission at short wavelengths. Furthermore, for shock velocities $\leqslant 150 \mathrm{~km} \mathrm{~s}^{-1}$, a small silicate grain signature is visible in the mid-infrared (9.7 and $18 \mu \mathrm{m}$ bands). This is due to a significant production of small silicate grains as a consequence of large silicate grain fragmentation.

In Figs. 17 and 18 we show the dust extinction (in the NIR-UV and IR wavelength regions respectively) for $V_{\text {shock }} \leqslant$ $175 \mathrm{~km} \mathrm{~s}^{-1}$. We notice that for all of the shock velocities, the characteristic UV bump at $217 \mathrm{~nm}$ disappears because of the destruction of the carriers, i.e. aromatic-rich hydrocarbon nanoparticles. Furthermore, we observe that, with increasing shock velocity, the UV extinction flattens primarily as a consequence of aromatic-rich nanoparticle destruction but also because of silicate grain destruction. On the other hand, the IR extinction shows small changes in shape. The large carbonaceous grain destruction is reflected by a drop in the $3-8 \mu$ m extinction, where carbonaceous grains give an important contribution to the total extinction.

\subsection{Dust destruction potential}

Comparing the pre-shock and post-shock size distributions we calculate the fraction of mass that has been destroyed during the shock for carbonaceous and silicate grains. Figure 19 shows the carbonaceous and silicate grain destruction (as a percentage) as a function of the shock velocity. Dotted and dashed lines show the relative contribution of sputtering and vapourisation to the total destruction (solid lines). We notice that for all of the shocks, the vapourisation play little role and that the destruction is dominated by the sputtering process. The shattering is not directly responsible for any dust destruction but only affects the shape of size distribution transferring mass from the large grains to the smaller ones, which are more susceptible to destruction.

5 The penetration depth, estimated with SRIM, for impinging $\mathrm{H}^{+}$ $\left(\mathrm{He}^{+}\right)$of energy $100(470) \mathrm{eV}$ (corresponding to the energy for a $200 \mathrm{~km} \mathrm{~s}^{-1}$ shock) in hydrogenated amorphous carbon is 4 (7) $\mathrm{nm}$. 


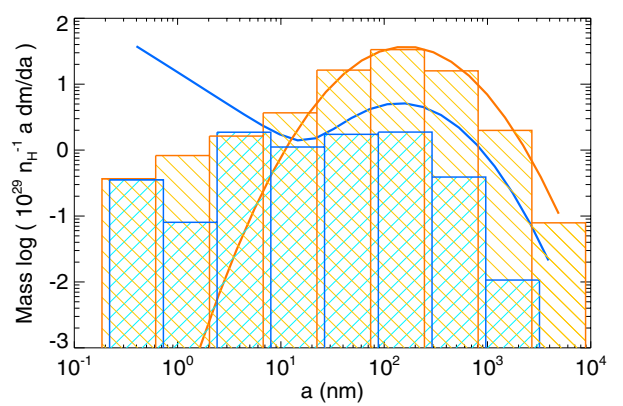

(a) $V_{\text {shock }}=50 \mathrm{~km} \mathrm{~s}^{-1}$

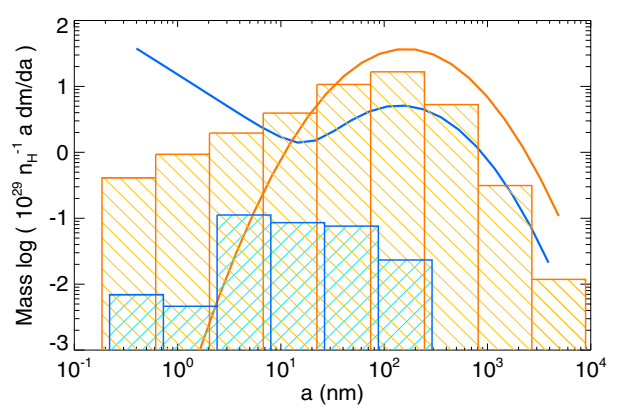

(d) $V_{\text {shock }}=125 \mathrm{~km} \mathrm{~s}^{-1}$

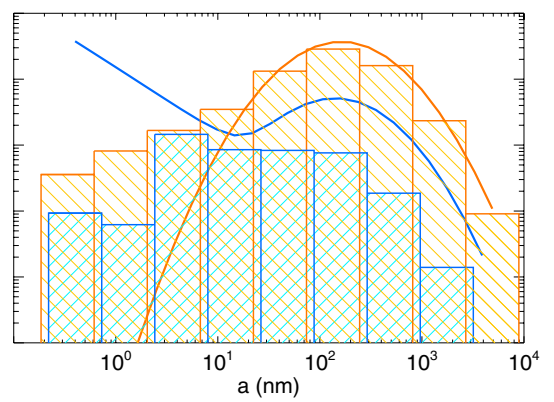

(b) $V_{\text {shock }}=75 \mathrm{~km} \mathrm{~s}^{-1}$

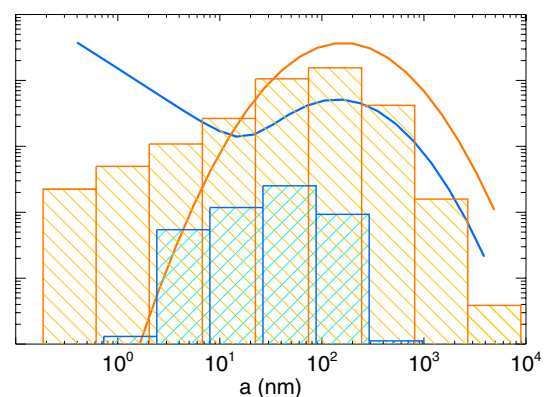

(e) $V_{\text {shock }}=150 \mathrm{~km} \mathrm{~s}^{-1}$

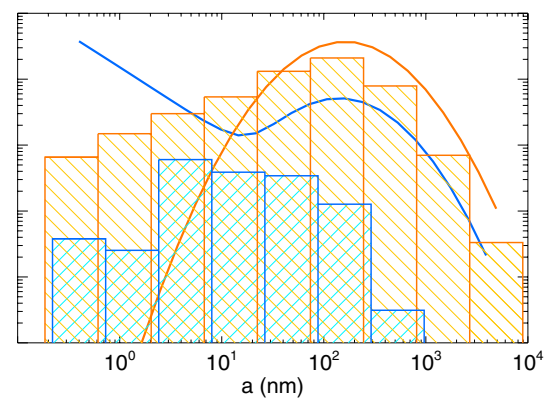

(c) $V_{\text {shock }}=100 \mathrm{~km} \mathrm{~s}^{-1}$

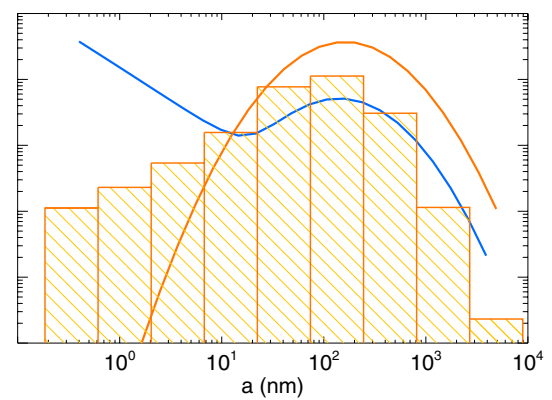

(f) $V_{\text {shock }}=175 \mathrm{~km} \mathrm{~s}^{-1}$

Fig. 15. Post-shock size distribution for different shock velocities. The post-shock distribution is divided into 9 bins while the solid lines represent the pre-shock distribution; carbonaceous (blue) and silicate (orange). The $200 \mathrm{~km} \mathrm{~s}^{-1}$ post-shock size distribution is almost identical to the case of a $175 \mathrm{~km} \mathrm{~s}^{-1}$ shock, and is therefore not shown here.

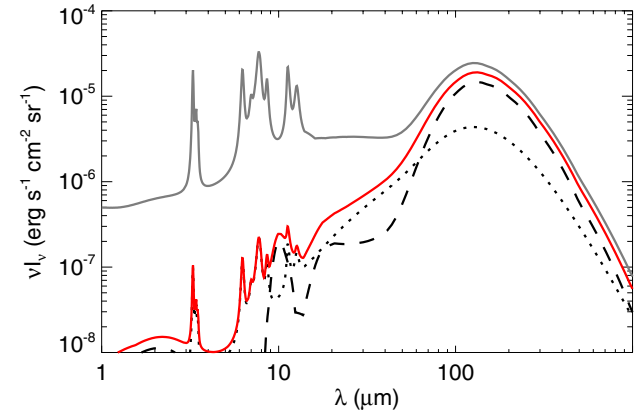

(a) $V_{\text {shock }}=50 \mathrm{~km} \mathrm{~s}^{-1}$

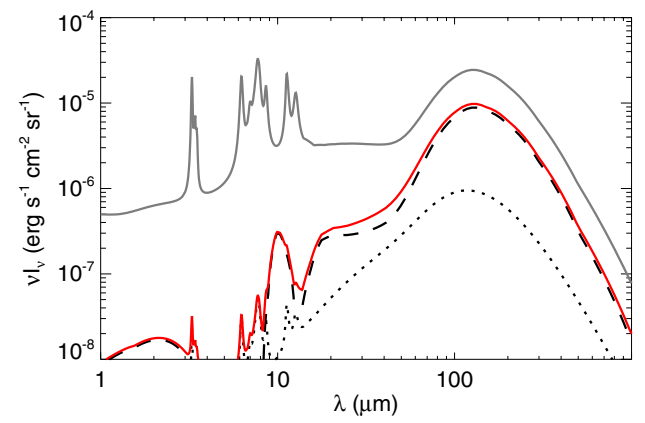

(d) $V_{\text {shock }}=125 \mathrm{~km} \mathrm{~s}^{-1}$

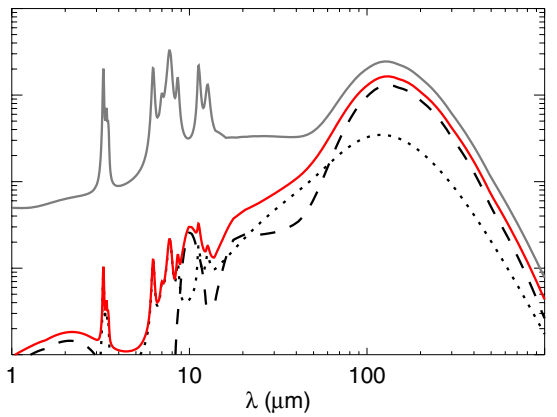

(b) $V_{\text {shock }}=75 \mathrm{~km} \mathrm{~s}^{-1}$

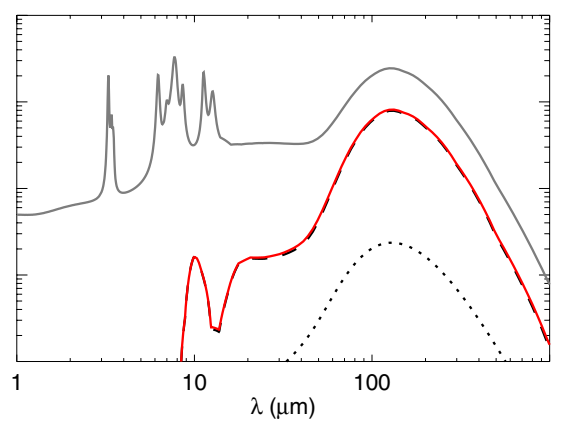

(e) $V_{\text {shock }}=150 \mathrm{~km} \mathrm{~s}^{-1}$



(c) $V_{\text {shock }}=100 \mathrm{~km} \mathrm{~s}^{-1}$

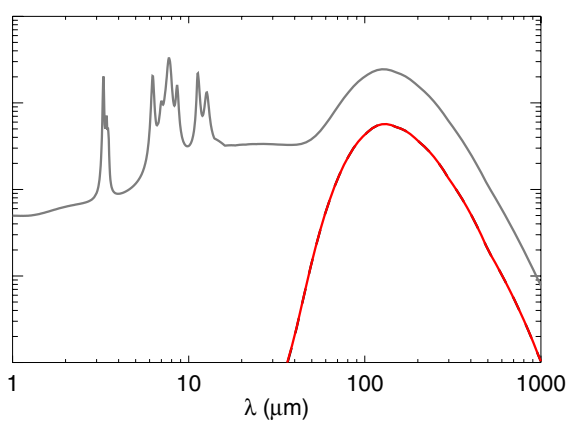

(f) $V_{\text {shock }}=175 \mathrm{~km} \mathrm{~s}^{-1}$

Fig. 16. Post-shock SED as a function of shock velocity. Grey lines indicate the pre-shock SED and red lines the post-shock SED. Dotted and dashed lines indicate the contribution of carbonaceous and silicate grains respectively, to the post-shock SED. The assumed column density is $N_{\mathrm{H}}=10^{20} \mathrm{~cm}^{-2}$. The $200 \mathrm{~km} \mathrm{~s}^{-1}$ case is almost identical to the case of a $175 \mathrm{~km} \mathrm{~s}^{-1}$ shock and is therefore not shown here.

In Table 6 we compare our dust destruction results with those of Jones et al. (1996) and Serra Díaz-Cano \& Jones (2008). In our study, both carbonaceous and silicate grains undergo more destruction than in the previous studies. This is mostly due to the adoption of the new dust model. In the Jones et al. (2013) dust model most of the carbonaceous mass is in small grains 
Marco Bocchio et al.: A re-evaluation of dust processing in supernova shock waves

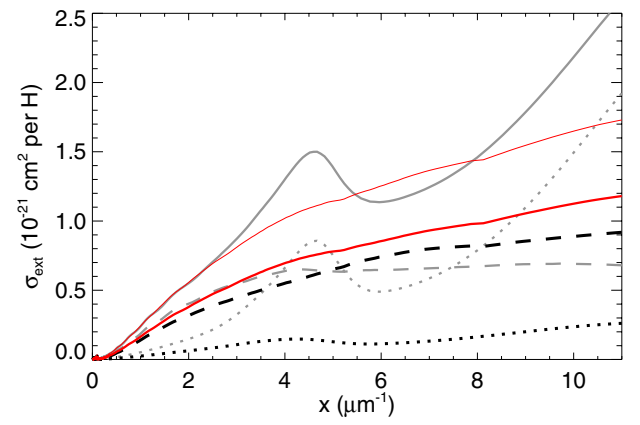

(a) $V_{\text {shock }}=50 \mathrm{~km} \mathrm{~s}^{-1}$

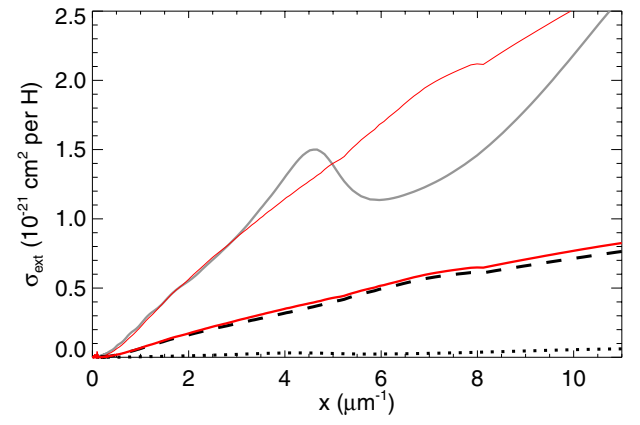

(d) $V_{\text {shock }}=125 \mathrm{~km} \mathrm{~s}^{-1}$

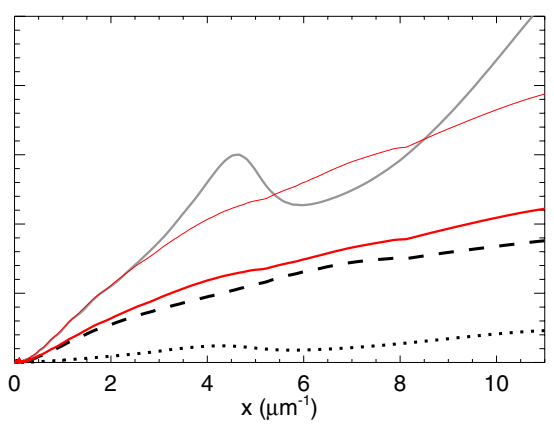

(b) $V_{\text {shock }}=75 \mathrm{~km} \mathrm{~s}^{-1}$



(e) $V_{\text {shock }}=150 \mathrm{~km} \mathrm{~s}^{-1}$

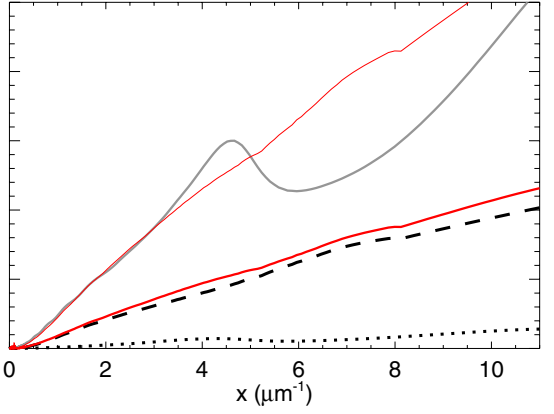

(c) $V_{\text {shock }}=100 \mathrm{~km} \mathrm{~s}^{-1}$

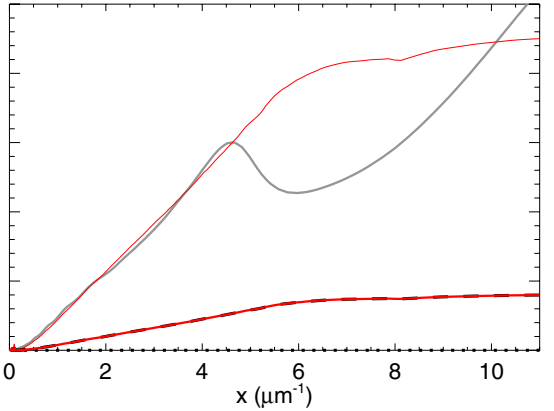

(f) $V_{\text {shock }}=175 \mathrm{~km} \mathrm{~s}^{-1}$

Fig. 17. Post-shock NIR-UV dust extinction as a function of shock velocity. The colour- and line style-coding are as per Fig. 16 . The 200 km $\mathrm{s}^{-1}$ case is almost identical to that of the $175 \mathrm{~km} \mathrm{~s}^{-1}$ shock and is therefore not shown here. The thin red lines show the post-shock extinction normalised to the $V$-band pre-shock extinction. For $V_{\text {shock }}=50 \mathrm{~km} \mathrm{~s}^{-1}$ the pre-shock dust extinction due to carbonaceous (grey dotted line) and silicate (grey dashed line) grains is shown.

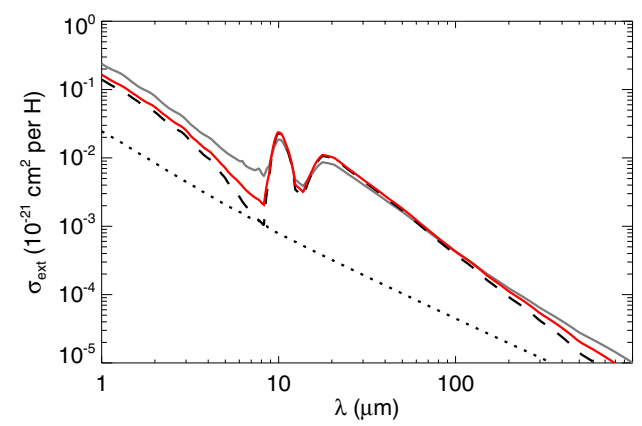

(a) $V_{\text {shock }}=50 \mathrm{~km} \mathrm{~s}^{-1}$



(d) $V_{\text {shock }}=125 \mathrm{~km} \mathrm{~s}^{-1}$

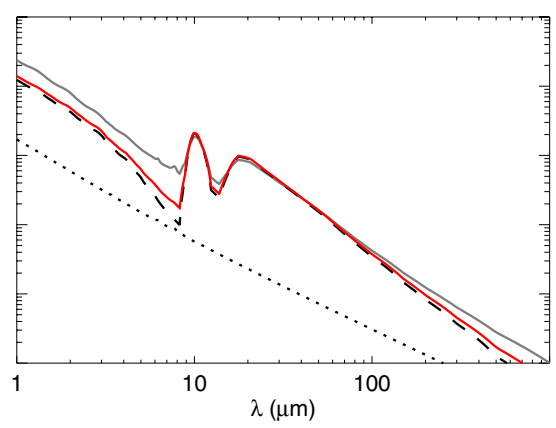

(b) $V_{\text {shock }}=75 \mathrm{~km} \mathrm{~s}^{-1}$

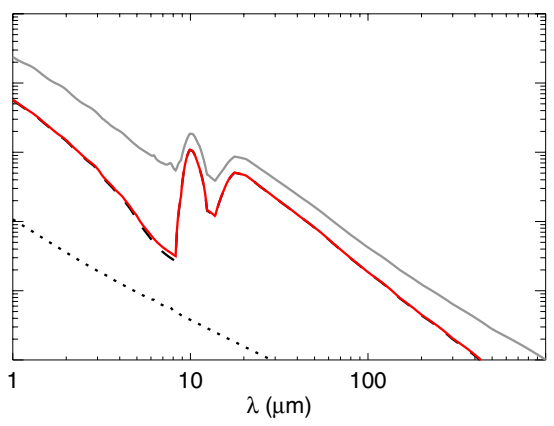

(e) $V_{\text {shock }}=150 \mathrm{~km} \mathrm{~s}^{-1}$

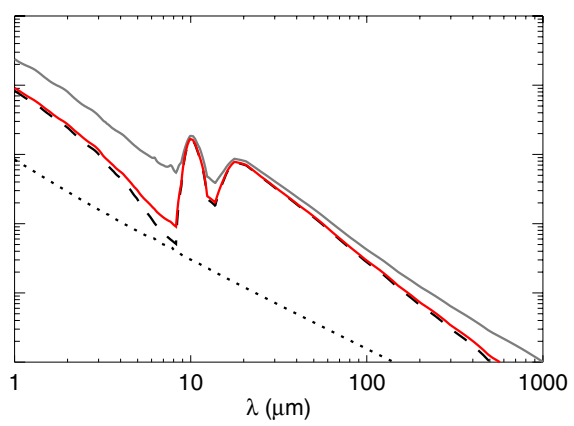

(c) $V_{\text {shock }}=100 \mathrm{~km} \mathrm{~s}^{-1}$

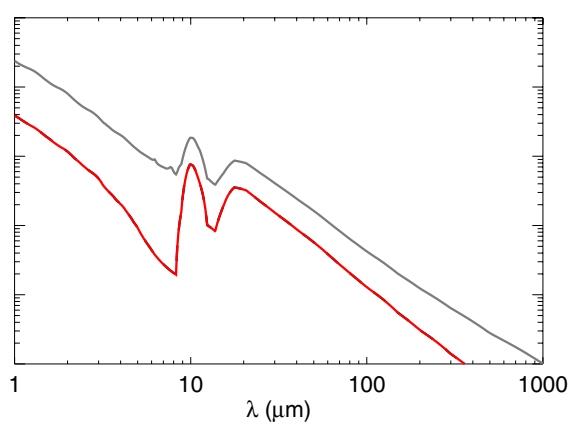

(f) $V_{\text {shock }}=175 \mathrm{~km} \mathrm{~s}^{-1}$

Fig. 18. Post-shock IR dust extinction as a function of shock velocity. Colour- and line style-coding are as per Fig. 16 . The $200 \mathrm{~km} \mathrm{~s}^{-1}$ case is almost identical to the case of a $175 \mathrm{~km} \mathrm{~s}^{-1}$ shock and is not shown here. 
Table 6. Dust destruction (percentage) as a function of the shock velocity, $V_{\text {shock}}$, for carbonaceous and silicate grains as estimated by JTH96 (Jones et al. 1996), SJ08 (Serra Díaz-Cano \& Jones 2008) and by this study.

\begin{tabular}{c|ccccccc}
\hline \hline$V_{\text {shock }}\left(\mathrm{km} \mathrm{s}^{-1}\right)$ & 50 & 75 & 100 & 125 & 150 & 175 & 200 \\
\hline \multicolumn{1}{l}{ carbonaceous grains } \\
\hline JTH96 & 1 & 5 & 7 & 13 & 12 & 21 & 47 \\
SJ08 & 4 & 21 & 31 & 56 & 58 & 81 & 100 \\
this study & 77 & 83 & 91 & 96 & 99 & 100 & 100 \\
\hline \multicolumn{2}{c}{ silicate grains } \\
\hline JTH96 & 2 & 12 & 18 & 33 & 32 & 41 & 49 \\
this study & 2 & 12 & 29 & 46 & 53 & 67 & 67 \\
\hline
\end{tabular}

Table 7. Dust destruction (percentage) for our standard $100 \mathrm{~km} \mathrm{~s}^{-1}$ shock, without and with the update of the ${ }^{(a)}$ charge scheme (from McKee et al. 1987 to WD01), ${ }^{(b)}$ the carbonaceous grain sputtering (from Tielens et al. 1994 to a-C(:H) sputtering), (c) the relative velocity distribution (from thermal and inertial sputtering to the skewed Maxwellian distribution).

\begin{tabular}{c|cc|cc}
\hline \hline & \multicolumn{2}{|c|}{ Previous } & \multicolumn{2}{c}{ Updated } \\
& "graphitic" & silicate & a-C:H & silicate \\
\hline charge scheme $^{a}$ & 90 & 28 & 91 & 29 \\
$\begin{array}{c}\text { a-C }(: H) \\
\text { sputtering }\end{array}$ & 5 & 28 & 91 & 29 \\
distribution $^{c}$ & 74 & 17 & 91 & 29 \\
\hline
\end{tabular}

( $a<20 \mathrm{~nm}$ ), which are rather fragile compared to larger grains (Micelotta et al. 2010a,b; Bocchio et al. 2012). Furthermore, the silicate grain distribution is almost mono modal and centred at a radius $a \sim 160 \mathrm{~nm}$, grains that have a large inertia and that are efficiently destroyed in shocks.

\subsection{Comparison with earlier results}

The introduction of more detailed physics into the GRASH_EX version of the original code leads to a re-evaluation of the dust destruction by SN shocks. The modifications to the dust physics that we have made can be summarised as follows:

- an update of the grain charge scheme from McKee et al. (1987) to WD01,

- an update of the carbonaceous grain material from graphitictype to a-C $(: \mathrm{H})$ materials,

- a unification of inertial and thermal sputtering with the use of the skewed Maxwellian distribution.

In order to understand the relative importance of the different updates, we compare the destruction for our standard shock with and without the introduction of each of the new features and report the results obtained in Table 7 . We notice that, while the change of the grain charge scheme little affects the results, updating the carbonaceous material and merging the thermal and inertial sputtering lead to increased dust destruction. In particular, we note that the lower density of the a-C(:H) particles with respect to graphitic-type materials makes them more susceptible to destructive processing. Finally, we point out that the displaced Maxwellian distribution better describes the physics of the interaction between gas ions and grains, with respect to the thermal/inertial approximation. We find that the inclusion of the more realistic physical treatment leads to the almost complete

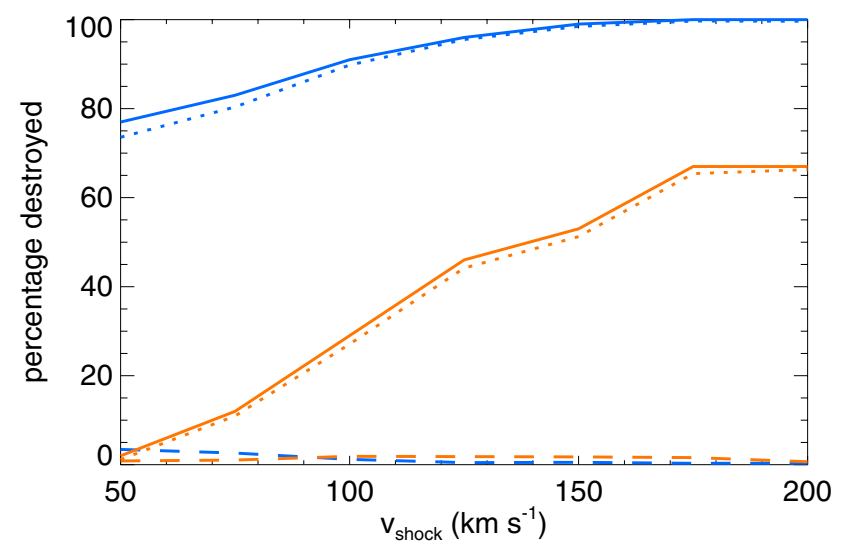

Fig. 19. Dust destruction (percentage) for carbonaceous grains (blue line) and silicate grains (orange line) as a function of the shock velocity. Dotted and dashed lines, respectively, represent the sputtering and vapourisation contributions to the destruction.

destruction of carbonaceous nanoparticles even in the case of a $50 \mathrm{~km} \mathrm{~s}^{-1}$ shock.

\subsection{Dust lifetime}

Following the approach by McKee (1989) we calculate the dust lifetime, $t_{\mathrm{SNR}}$ as

$t_{\mathrm{SNR}}=\frac{\tau_{\mathrm{SN}} M_{\mathrm{ISM}}}{\int \epsilon\left(v_{\mathrm{S}}\right) \mathrm{d} M_{\mathrm{S}}\left(v_{\mathrm{S}}\right)}$

where $\tau_{S N}=125 \mathrm{yr}$ is the effective interval between $\mathrm{SNe}$, $M_{\text {ISM }}=4.5 \times 10^{9} M_{\odot}$ is the total interstellar gas and dust mass in the Galaxy, $v_{\mathrm{s}}$ is the shock velocity, $\epsilon\left(v_{\mathrm{S}}\right)$ is the efficiency of grain destruction and $M_{\mathrm{s}}\left(v_{\mathrm{s}}\right)$ is the mass of the gas shocked to at least $v_{\mathrm{s}}$ by a $\mathrm{SN}$ remnant in the Sedov-Taylor stage. Following Jones et al. (1994) we have

$M_{\mathrm{s}}\left(v_{\mathrm{s} 7}\right)=6800 E_{51} / v_{\mathrm{s} 7}^{2} M_{\odot}$,

where $E_{51}$ is the SN energy in units of $10^{51} \mathrm{erg}$ and $v_{\mathrm{s} 7}$ the shock velocity in units of $100 \mathrm{~km} \mathrm{~s}^{-1}$. Then assuming, as per McKee \& Ostriker (1977), a ratio of warm to hot ISM filling factor of $f_{\mathrm{w}} / f_{\mathrm{h}}=0.3 / 0.7=0.43$ and a mean SN energy of $10^{51}$ ergs we obtain

$t_{\mathrm{SNR}}=\frac{9.7 \times 10^{7}}{\int \epsilon\left(v_{\mathrm{s} 7}\right) / v_{\mathrm{s} 7}^{3} \mathrm{~d} v_{\mathrm{s} 7}} \mathrm{yr}$.

We then use Eq. (4) from McKee (1989) to determine the fraction of the elements locked up in the surviving grains, $\delta_{\mathrm{eq}}$

$\delta_{\text {eq }}=\delta_{\text {in }}\left[1+\frac{t_{\text {in }}}{t_{\text {SNR }}}\right]^{-1}$,

where $t_{\text {in }}$ is the timescale for the injection of dust into the ISM and $\delta_{\text {in }}$ is the fraction of material injected in the ISM initially contained in the dust (we assume $\delta_{\text {in }}=0.9$ ). We can approximate the carbonaceous destruction efficiency as:

$\epsilon\left(v_{\mathrm{s} 7}\right)= \begin{cases}0.66+0.23 v_{\mathrm{s} 7} & \text { for } 0.5<v_{\mathrm{s} 7} \leqslant 1.5 \\ 1 & \text { for } 1.5<v_{\mathrm{s} 7}<2.0\end{cases}$

and for silicate grains:

$\epsilon\left(v_{\mathrm{s} 7}\right)= \begin{cases}0.61 v_{\mathrm{s} 7}-0.31 & \text { for } 0.5<v_{\mathrm{s} 7} \leqslant 1.25 \\ 0.11+0.28 v_{\mathrm{s} 7} & \text { for } 1.25<v_{\mathrm{s} 7} \leqslant 2\end{cases}$ 
Assuming the uncertainties estimated by Jones \& Nuth (2011), this leads to a dust lifetime of:

$t_{\mathrm{SNR}}= \begin{cases}(6.2 \pm 5.6) \times 10^{7} \mathrm{yr} & \text { for carbonaceous grains } \\ (3.1 \pm 2.7) \times 10^{8} \mathrm{yr} & \text { for silicate grains }\end{cases}$

While the estimate for silicate grains is about the same as in the previous study by Jones et al. (1996), the estimate for carbonaceous grains is an order of magnitude shorter than in that study and a factor $\sim 3$ shorter than in the work by Serra Díaz-Cano \& Jones (2008).

If we assume that $t_{\text {in }} \sim(3 \pm 1.5) \times 10^{9} \mathrm{yr}$ as estimated by different groups (Dwek \& Scalo 1980; Gehrz 1989; Jones \& Tielens 1994), the dust destruction timescale is much shorter than the timescale for its injection into the ISM. The equilibrium fraction of elements locked in grains, in the absence of dust re-formation in the ISM, is then:

$\delta_{\text {eq }}= \begin{cases}0.018 \pm 0.016 & \text { for carbonaceous grains } \\ 0.08 \pm 0.07 & \text { for silicate grains }\end{cases}$

Comparing these values with typical values for the diffuse ISM (i.e. $\geq 50 \%(\geq 90 \%)$ of $\mathrm{C}(\mathrm{Si}, \mathrm{Fe}$ and $\mathrm{Mg}$ ) atoms locked into grains), we see that our estimate is far from consistent with the observed values and we cannot avoid the conclusion that dust must be efficiently reformed in the ISM (see discussion in Sect. 5.5).

\section{Discussion}

The use of the Jones et al. (2013) dust model, with the majority of the carbonaceous dust mass in small particles, coupled with the detailed physics of a-C(:H) material processing in energetic regions (Serra Díaz-Cano \& Jones 2008; Micelotta et al. 2010a,b; Bocchio et al. 2012, 2013), leads to a very short carbon dust cycling timescale in the ISM. Our modelling therefore predicts that the observed fraction of stardust material locked into carbonaceous grains in the ISM is essentially zero $\left(\delta_{\mathrm{eq}}=\right.$ $0.018 \pm 0.016)$ and that the a-C $(: \mathrm{H})$ grain "lifetime" is very short $\left(\approx[6.2 \pm 5.6] \times 10^{7} \mathrm{yr}\right)$. Our carbonaceous dust lifetime estimation is almost three times shorter than the previous estimate by Serra Díaz-Cano \& Jones (2008). This is due to the introduction of the molecular approach developed by Micelotta et al. (2010a), which leads to a high destruction rate for small grains. Furthermore, merging the inertial and thermal sputtering into a single process, leads to a lifetime three times shorter than that estimated by Micelotta et al. (2010a). This is because of the effects of the "high-temperature tail" in the skewed Maxwellian velocity distribution (see Fig. 6). The very large discrepancy (by a factor of about 50) between the derived carbonaceous dust lifetime and its formation timescale around evolved stars $\left(\simeq 3 \times 10^{9} \mathrm{yr}\right)$ then implies that most of the carbonaceous dust in the ISM must have been re-formed there, most probably by the accretion of $\mathrm{C}$ (and $\mathrm{H}$ ) atoms and small hydrocarbon species onto the more resilient silicate grains and that this must occur in dense regions of the ISM (Jones et al. 2013, 2014). Chemical vapour deposition (CVD) is a long-used process for hydrogenated amorphous carbon formation in the laboratory (e.g., Robertson 1986). Thus, and given that much of the silicate dust then survives in the ISM, there is then a viable mechanism for the re-formation of carbonaceous grain mantles via a CVD-type accretion process in the dense ISM.

On the other hand, the long coupling time between the gas and large silicate grains in a shock leads to high silicate destruction rates and we find that only a fraction of $\delta_{\text {eq }}=0.08 \pm 0.07$ of silicate stardust could be preserved in the ISM with a "lifetime" of $\approx 3.1 \pm 2.7 \times 10^{8} \mathrm{yr}$, which is about an order of magnitude shorter than the injection timescale. However, as argued by Jones \& Nuth (2011), the uncertainties on these calculations are of the order of the $100 \%$. Moreover, despite the introduction of much new physics into the code, the exact nature of some of the physical processes is still rather uncertain. In particular, in this section we will focus on the silicate core sputtering, the dynamics of the collision between large silicate grains and carbonaceous nanoparticles, the structure of the shock profile and the mass binning of the dust size distribution.

\subsection{Silicate core sputtering}

In this study, as per Jones et al. (1994, 1996), we model the silicate grain sputtering due to collisions with ions using the estimates by Tielens et al. (1994). However, more experimental data are now available and the sputtering yield for silicate grains can be estimated with more precision at low energies. The SRIM software (Ziegler et al. 1985) provides a means to calculate (to within $4 \%$ of the experimental values) the sputtering yield due to the interaction between ions and silicate target. In order to calculate the sputtering yield, the user must supply the following key parameters:

- the material density, $\rho$,

- the surface binding energy, $E_{\mathrm{S}}$, i.e. the energy that binds a surface atom to the lattice,

- the lattice binding energy, $E_{\mathrm{B}}$, i.e. the energy that binds an atom to a lattice site within the bulk, and,

- the displacement energy, $E_{\mathrm{D}}$, i.e. the energy needed to displace an atom within the lattice.

May et al. (2000) estimated these parameters for forsterite-type materials, $(\mathrm{Fe}, \mathrm{Mg})_{2} \mathrm{SiO}_{4}$. In particular, they used the heat of atomisation as an estimate of the surface binding energy and performed calculations within the framework of the ionic model in order to quantify the lattice binding energy. However, while the surface and lattice binding energy are experimentally or theoretically rather well constrained, the value of the displacement energy is still uncertain. May et al. (2000) took the displacement energy of $\mathrm{Mg}$ and $\mathrm{O}$ atoms in $\mathrm{MgO}$ compounds as a guide ( $E_{\mathrm{D}}=52$ and $54 \mathrm{eV}$ for $\mathrm{Mg}$ and $\mathrm{O}$ respectively) and assumed $E_{\mathrm{D}}=50 \mathrm{eV}$ for all the atoms in the silicate. However, the uncertainty on this parameter may be a factor of two and, for example, Wang et al. (1999) assumed a displacement energy of $E_{\mathrm{D}} \approx 25 \mathrm{eV}$ for similar forsterite-type materials.

Experimental studies show that, under the effect of energetic ion irradiation, silicate grains become amorphous in structure (Demyk et al. 2001; Carrez et al. 2002) and so the displacement energy will be lower than for a crystalline structure. We therefore adopt a displacement energy of $E_{\mathrm{D}}=25 \mathrm{eV}$. Furthermore, since in an amorphous silicate, $\mathrm{Fe}$ and $\mathrm{Mg}$ atoms will tend to have no preferred sites, we assume the same values for the lattice binding energy for both atoms $\left(E_{\mathrm{B}} \approx 1.9 \mathrm{eV}\right)$. We estimate the surface binding energy with the same approach as per May et al. (2000) and we assume the same silicate density as in the Jones et al. (2013) dust model. We report the assumed values in Table 8.

We run SRIM for different impact angles and energies for $\mathrm{H}^{+}$and $\mathrm{He}^{+}$projectiles, simulating $10^{6}$ collisions in each case. Assuming isotropic collisions, we calculate the average sputtering yield, for each target atom, at a given incident energy and 
Table 8. Forsterite-type silicate physical properties.

\begin{tabular}{c|ccccc}
\hline \hline Material & Element & $\rho^{a}$ & $E_{\mathrm{D}}{ }^{b}$ & $E_{\mathrm{B}}{ }^{c}$ & $E_{\mathrm{S}}{ }^{c}$ \\
\hline \multirow{4}{*}{ forsterite-type } & $\mathrm{Si}$ & & 25 & 4 & 6.8 \\
& $\mathrm{O}$ & & 25 & 15.5 & 4.75 \\
& $\mathrm{Fe}$ & 2.5 & 25 & 1.9 & 6.47 \\
& $\mathrm{Mg}$ & & 25 & 1.9 & 3.7 \\
\hline
\end{tabular}

Notes. $\rho$ is the material density, $E_{\mathrm{D}}, E_{\mathrm{B}}$ and $E_{\mathrm{S}}$ are the displacement energy, the lattice binding energy and the surface binding energy, respectively. ${ }^{(a)}$ We adopt the same density as per Jones et al. (2013). ${ }^{(b)}$ Wang et al. (1999). ${ }^{(c)}$ Adapted values from May et al. (2000).

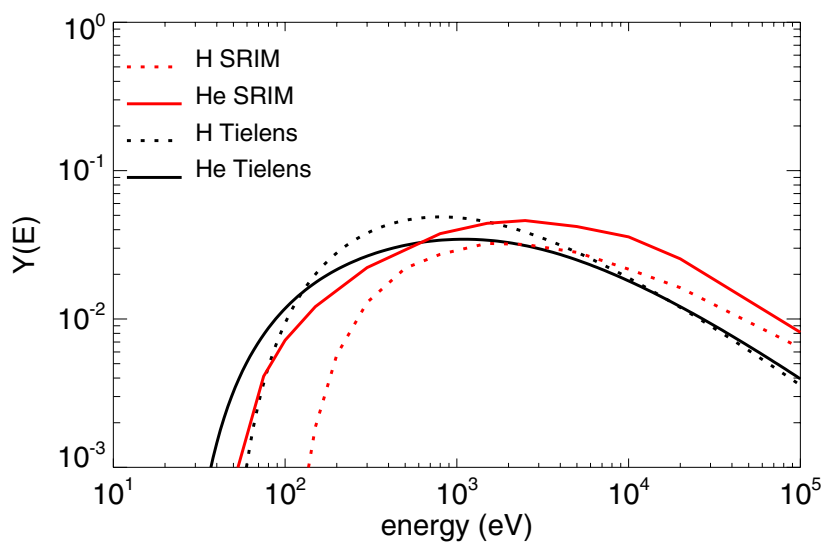

Fig. 20. Silicate sputtering yield for incident $\mathrm{H}^{+}$(dotted lines) and $\mathrm{He}^{+}$ (solid lines) as a function of the impinging energy. Tielens et al. (1994) estimates (black lines) and SRIM calculations (red lines) are shown. The sputtering yield for impinging $\mathrm{He}^{+}$ions are multiplied by their relative abundance with respect to hydrogen, i.e. $X_{\mathrm{He}}=0.1$.

integrate over all the impact angles

$Y_{i}(E)=\int_{0}^{\pi / 2} \mathrm{~d} \theta Y_{i}(\theta, E) \sin (\theta)$,

where $Y(\theta, E)$ is the sputtering yield at a given angle, $\theta$, and energy, $E$, for the $i$ th target atom. Then, averaging over all the different target atoms, the sputtering yield is given by

$\overline{Y(E)}=\sum_{i} Y_{i}(E) w_{i}$

where $w_{i}=\frac{m_{i}}{\sum_{j} m_{j}}$ and $m_{i}$ the $i$ th ion mass.

In Fig. 20 we compare the silicate sputtering yield as estimated by Tielens et al. (1994) and as calculated using SRIM for $\mathrm{H}^{+}$and $\mathrm{He}^{+}$impinging projectiles. We note that, the threshold energy is shifted to higher energies with respect to the Tielens et al. (1994). However, the SRIM results are sensitive to the assumed value of the displacement energy and a higher displacement energy (e.g. $E_{\mathrm{D}}=50 \mathrm{eV}$ as estimated by May et al. 2000) will lead to a higher threshold energy and a lower sputtering yield over a large range of energies (Leroux, priv. comm.). In any case, these calculations seem to indicate that the estimates by Tielens et al. (1994) represent an upper limit and that the silicate destruction in shocks may be significantly lower.

Running GRASH_EX for the standard shock $\left(v_{\text {shock }}=\right.$ $100 \mathrm{~km} \mathrm{~s}^{-1}$ ) and replacing the Tielens et al. (1994) approach with the silicate sputtering yield derived using the SRIM simulations (with the assumed parameters in Table 8) we obtain a silicate destruction of $14 \%$, which is almost half of the destruction obtained with the Tielens et al. (1994) yields. This result is even



Fig. 21. Percentage of the total dust mass affected by shattering in graingrain collisions as a function of the shock velocity.

more dramatic if we assume a displacement energy of $50 \mathrm{eV}$ : the silicate destruction in this case is only $6 \%$.

\subsection{Shattering and vapourisation}

In Sect. 4 we showed that grain-grain collisions trigger the fragmentation of large grains leading to a broadening of the dust size distribution (see Fig. 15b). We have estimated the fraction of mass which is affected by shattering for different shock velocities and show the results in Fig. 21. We note that for carbonaceous grains the shattering is not very important $(\lesssim 10 \%$ of the mass affected) while for silicate grains the affected mass peaks at a value of $\sim 35 \%$ for a shock velocity of $100 \mathrm{~km} \mathrm{~s}^{-1}$ (i.e. where the betatron acceleration is most efficient). Since the size distribution is dominated by small carbonaceous grains and large silicate grains, grain-grain collisions mainly occur between these two populations. In our study the dynamics of small carbon grain impacts on silicates are treated using a "hard sphere" approach (Jones et al. 1996). However, small a-C(:H) grains are low density, open structures and will therefore act as "soft" impactors, rather than hard spheres. For example, a molecular dynamics study on collisions between fullerenes $\mathrm{C}_{60}$ (which have a rather resistant and stable configuration) and graphite surfaces show that up to energies of $6 \mathrm{keV}$ both the projectile and the target are little affected by the collision (Smith \& Webb 1993). This range of energies is typical for collisions between small and large grains in $50 \mathrm{~km} \mathrm{~s}^{-1}$ shocks suggesting that our calculation of the cratering-type erosion of large amorphous silicate and carbonaceous grains by small a-C(:H) particle collisions, and their subsequent evaporation, are severely over-estimated. In order to study "soft" molecule impacts on "hard" surfaces dedicated molecular dynamics modelling will be required, which is clearly beyond the scope of this paper.

In order to estimate the effects of reduced shattering by nanoparticle impacts we run the $100 \mathrm{~km} \mathrm{~s}^{-1}$ shock ignoring the shattering process. Figure 22 shows the post-shock size distribution in this case. Comparing the size distribution with and without the inclusion of the shattering process we note that shattering is the process responsible for the broadening of the size distribution. However, we note that the effect of the shattering process is to re-distribute the dust mass across the bins but that it does not lead to any net mass loss. On the other hand, the redistribution of the dust mass leads to a second-order effect on the dust destruction. Ignoring the shattering process, the silicate grain destruction is slightly lower $(\sim 26 \%)$ than in the case where 


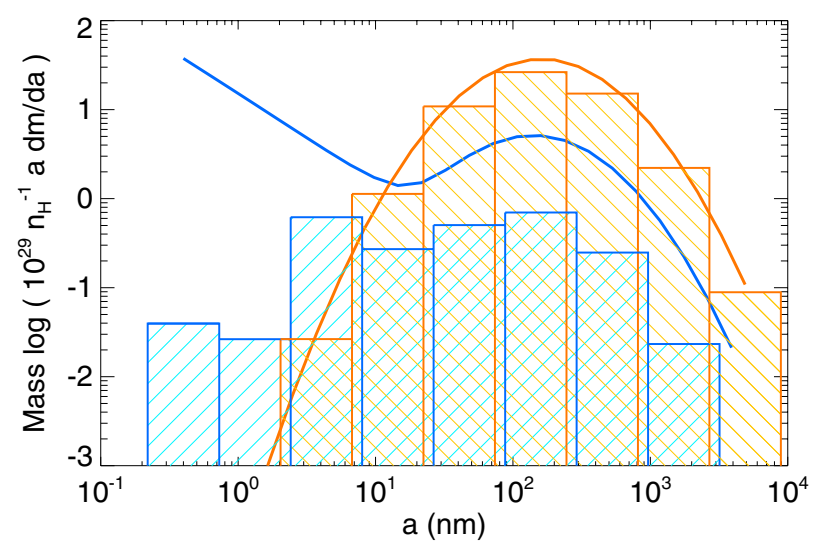

Fig. 22. Size distribution for the case of $100 \mathrm{~km} \mathrm{~s}^{-1}$ with no shattering.

this process is included ( $\sim 29 \%)$, while it does not lead to any change for carbonaceous grains. We also note that with shattering switched off the log-normal shape of the amorphous silicate size distribution is preserved and that the log-normal distribution of the large carbonaceous grains is also somewhat preserved.

\subsection{Steady state shock vs. hydro-dynamical calculations}

The approach that we have adopted here, as per Jones et al. (1994, 1996), is to assume steady state shocks propagating through the inter-cloud medium. However, full time-dependent hydro-dynamical modelling is really required to better evaluate dust processing in evolving $\mathrm{SN}$ remnants propagating through the ISM. Preliminary, hydro-dynamical studies indicate that the dust destruction effects could be reduced by about $20 \%$ but, more importantly, show that the shock velocity probability distribution appears to decrease to almost zero at $130-150 \mathrm{~km} \mathrm{~s}^{-1}$ and is negligible for higher velocities (Slavin, in prep.). Thus, physically-realistic studies of dust processing in SN shocks in the ISM strongly implies that our dust destruction estimates are rather upper limits.

\subsection{Accuracy and reliability of the simulations}

For all of the simulations we run we assume a pre-shock size distribution divided into 9 bins. However, in this way we could in principle introduce errors into the resulting dust SED and extinction compared to the case of a division of the size distribution into an infinite number of bins. We want to check whether a less accurate description of the size distribution can affect the dust emission and extinction. We divided the pre-shock size distribution into 9, 15 and 25 bins and we calculate with DustEM the emission and extinction in the three cases.

First of all we compare the IR emission for the fine binning with respect to a coarser one. We calculate the difference in intensity for each wavelength $\left(d_{n, m}\right.$, with $n$ and $m$ the number of bins in the two cases) between the two cases and we normalise it to the intensity calculated with 25 bins

$$
d_{25,9}=\left|I_{v, 25}-I_{v, 9}\right| / I_{v, 25}
$$

$d_{25,15}=\left|I_{v, 25}-I_{v, 15}\right| / I_{v, 25}$,

where $I_{v, \mathrm{n}}$ is the dust emission calculated using $n$ bins. The error that we introduce by dividing the size distribution into less than 25 bins can be as large as $100 \%$ of the emission. We then interpolate the coarser binning size distributions into 25 bins in order to mimic a finer grid and recalculate the difference in emission.
The error we make, even assuming a grid of 9 bins (but interpolating to 25 bins), is never larger than $\sim 20 \%\left(d_{25,15} \lesssim 8 \%\right.$ interpolating to 25 bins) across the whole range of wavelengths. For our runs we can therefore use 9 bins and interpolate the resulting size distribution onto a 25 bin grid. This allows us to spend less computation time and to still produce reliable results.

We follow the same procedure for the extinction in the NIRUV and IR bands. We define $d_{\mathrm{n}, \mathrm{m}}^{\text {ext,UV }}$ and $d_{\mathrm{n}, \mathrm{m}}^{\text {ext,IR }}$ the normalised difference between the extinction in the case of $n$ and $m$ bins in the NIR-UV and IR bands respectively. Also in this case the interpolation gives a good and reliable alternative to a finer grid ( $d_{25 \text {, } \mathrm{m}} \lesssim 1 \%$, for $m=9$ and 15 , interpolation onto a 25 bin grid).

\subsection{Consequences of the uncertainties}

Using the silicate core sputtering estimates by Tielens et al. (1994), the lifetime of silicate grains is almost an order of magnitude shorter than the injection timescale. This leads us to the conclusion that, in order to have a fraction of heavy elements locked in grains comparable to the observed values, we need to allow for the re-formation of silicate grains in the ISM. However, as pointed out by Jones \& Nuth (2011), silicate re-formation under the low density and temperature conditions of the ISM seems to be a rather problematic scenario. However, $\mathrm{SiO}$ and silicate grain formation at low temperatures ( $T \lesssim 15 \mathrm{~K}$ ) has been observed in the laboratory using idealised neon matrix and helium droplet isolation techniques (Krasnokutski et al. 2014; Rouillé et al. 2014). The oligomerisation of $\mathrm{SiO}$ molecules is barrierless and therefore could be an efficient mechanism for silicate formation in the low-temperature ISM (Rouillé et al. 2014).

SRIM calculations seem to indicate that the silicate sputtering rate might be significantly lower than estimated by Tielens et al. (1994) therefore leading to a much longer silicate grain lifetime $\left(t_{\mathrm{SNR}} \geqslant 6 \times 10^{8} \mathrm{yr}\right)$, possibly of the same order as the injection timescale. This would then imply a totally different scenario for silicate grain evolution and therefore make the previouslyassumed need to re-form silicate dust in the ISM no longer a requirement for new dust models.

\section{Conclusions}

In this study we have revisited the long-standing question of dust processing in interstellar shocks and the discrepancy with the dust injection timescale. Updating the GRASH code presented by Jones et al. $(1994,1996)$ we have re-evaluated dust destruction in shocks with velocities between 50 and $200 \mathrm{~km} \mathrm{~s}^{-1}$. In particular, we use the grain size distribution and material compositions of the Jones et al. (2013) dust model. Furthermore, we better model the dynamics of grains in the shock by updating the grain charge scheme with that of WD01 and by merging the inertial and thermal sputtering into a single process. Moreover, we have coupled the GRASH_EX code to the DustEM code in order to give the possibility to define any size distribution and to calculate the post-shock dust emission and extinction in shocked regions.

As a result of the new carbonaceous material and by putting most of the carbonaceous mass in the smallest grains, carbon dust is efficiently destroyed, even in a $50 \mathrm{~km} \mathrm{~s}^{-1}$ shock, which leads to a short carbon grain lifetime $\left(t_{\mathrm{SNR}, \mathrm{c}}=[6.2 \pm 5.6] \times\right.$ $10^{7} \mathrm{yr}$ ). Silicate grains are more resilient and large grains survive even in fast shocks. However, the calculated silicate grain lifetime, despite being longer than the carbonaceous grain lifetime is still short $\left(t_{\mathrm{SNR}, \mathrm{s}}=[3.1 \pm 2.7] \times 10^{8} \mathrm{yr}\right)$ compared to the 
dust injection timescale $\left(t_{\text {in }}=3 \times 10^{9} \mathrm{yr}\right.$ ), leading to a predicted low fraction of elements locked in grains $\left(\delta_{\text {eq,s }}=0.08 \pm 0.07\right)$.

The calculated fraction of elements locked in grains is not compatible with the observed values (i.e. $\geqslant 50 \%$ for $\mathrm{C}$ and $\geqslant 90 \%$ for $\mathrm{Si}, \mathrm{Fe}$ and $\mathrm{Mg}$ ). This implies that the re-formation of grains in denser regions of the interstellar medium must be efficient. While, there seems to be viable mechanisms for the re-formation of carbonaceous grains from the gas phase under low temperature and pressure conditions (e.g. Dartois et al. 2005), silicate grains are seemingly hard to re-form in the dense ISM and must therefore be formed in AGB stars and preserved in interstellar shocks (Jones \& Nuth 2011). This study is therefore not able to solve this long-standing conundrum. Although recent experiments (Krasnokutski et al. 2014; Rouillé et al. 2014) show that it may be possible to form silicates under ISM conditions.

However, it seems that the modelling of silicate sputtering together with hydrodynamical simulations of shock dynamics are absolutely critical to advance our understanding and will possibly give lower silicate sputtering resulting in a longer lifetime $t_{\mathrm{SNR}, \mathrm{s}} \geqslant 6 \times 10^{8} \mathrm{yr}$. This could lead to a different scenario, making the assumed need to re-form the silicate dust in the ISM no longer such a stringent requirement. These features must therefore be explored more in detail in future studies.

Finally, in the recent Jones et al. (2013) dust model small carbonaceous grains and large silicate grains are the most abundant species, therefore a correct treatment of the interaction between them is necessary in order to have a better estimate of the fragmentation of the large silicate grains. Molecular dynamics simulations (e.g. Smith \& Webb 1993) seem to indicate that this process would be much less effective than in the case of hard spheres collisions therefore leading to less mass in small silicate grains and reduced silicate emission in the MIR.

\section{References}

Arendt, R. G., Dwek, E., Kober, G., Rho, J., \& Hwang, U. 2014, ApJ, 786, 55 Barlow, M. J. 1978a, MNRAS, 183, 397

Barlow, M. J. 1978b, MNRAS, 183, 367

Bocchio, M., Jones, A. P., Verstraete, L., et al. 2013, A\&A, 556, A6

Bocchio, M., Micelotta, E. R., Gautier, A.-L., \& Jones, A. P. 2012, A\&A, 545, A124

Carrez, P., Demyk, K., Cordier, P., et al. 2002, Meteorit. Planet. Sci., 37, 1599

Compiègne, M., Verstraete, L., Jones, A., et al. 2011, A\&A, 525, A103

Cowie, L. L. 1978, ApJ, 225, 887

Dartois, E., Muñoz Caro, G. M., Deboffle, D., Montagnac, G., \& D’Hendecourt, L. 2005, A\&A, 432, 895

Demyk, K., Carrez, P., Leroux, H., et al. 2001, A\&A, 368, L38

Draine, B. T. 1981, ApJ, 245, 880

Draine, B. T., \& Li, A. 2001, ApJ, 551, 807

Draine, B. T., \& Li, A. 2007, ApJ, 657, 810

Draine, B. T., \& Salpeter, E. E. 1979a, ApJ, 231, 438
Draine, B. T., \& Salpeter, E. E. 1979b, ApJ, 231, 77

Dwek, E., \& Scalo, J. M. 1980, ApJ, 239, 193

Gehrz, R. 1989, in Interstellar Dust, eds. L. J. Allamandola, \& A. G. G. M. Tielens, IAU Symp., 135, 445

Guillet, V. 2008, Évolution des poussières dans les chocs, Ph.D. thesis

Hoppe, P., Leitner, J., Meyer, B. S., et al. 2009, ApJ, 691, L20

Hoppe, P., Leitner, J., Gröner, E., et al. 2010, ApJ, 719, 1370

Jenkins, E. B. 2009, ApJ, 700, 1299

Jones, A. P. 2012a, A\&A, 540, A1

Jones, A. P. 2012b, A\&A, 540, A2

Jones, A. P. 2012c, A\&A, 542, A98

Jones, A. P. 2012d, A\&A, 545, C2

Jones, A. P. 2012e, A\&A, 545, C3

Jones, A. P. 2013, Planet. Space Sci., 100, 26

Jones, A. P., \& Nuth, J. A. 2011, A\&A, 530, A44

Jones, A. P., \& Tielens, A. G. G. M. 1994, in The Diffuse Interstellar Bands, ed. A. G. G. M. Tielens, 79

Jones, A. P., Tielens, A. G. G. M., Hollenbach, D. J., \& McKee, C. F. 1994, ApJ, 433, 797

Jones, A. P., Tielens, A. G. G. M., \& Hollenbach, D. J. 1996, ApJ, 469, 740

Jones, A. P., Fanciullo, L., Köhler, M., et al. 2013, A\&A, 558, A62

Jones, A. P., Ysard, N., Köhler, M., et al. 2014, Faraday Discussions, accepted

Jurac, S., Johnson, R. E., \& Donn, B. 1998, ApJ, 503, 247

Krasnokutski, S. A., Rouillé, G., Jäger, C., et al. 2014, ApJ, 782, 15

Li, A., \& Draine, B. T. 2001, ApJ, 554, 778

Lodders, K. 2006, ApJ, 647, L37

Mathis, J. S., Rumpl, W., \& Nordsieck, K. H. 1977, ApJ, 217, 425

Matsuura, M., Dwek, E., Meixner, M., et al. 2011, Science, 333, 1258

May, P. W., Pineau des Forêts, G., Flower, D. R., et al. 2000, MNRAS, 318, 809

McKee, C. 1989, in Interstellar Dust, eds. L. J. Allamandola, \& A. G. G. M. Tielens, IAU Symp., 135, 431

McKee, C. F., \& Ostriker, J. P. 1977, ApJ, 218, 148

McKee, C. F., Hollenbach, D. J., Seab, G. C., \& Tielens, A. G. G. M. 1987, ApJ, 318,674

McQueen, R. G., Marsh, S. P., Taylor, J. W., Fritz, J. N., \& Carter, W. J. 1970, High-Velocity Impact Phenomenae, ed. R. Kinslow (New York: Academic) Micelotta, E. R., Jones, A. P., \& Tielens, A. G. G. M. 2010a, A\&A, 510, A36

Micelotta, E. R., Jones, A. P., \& Tielens, A. G. G. M. 2010b, A\&A, 510, A37

Micelotta, E. R., Jones, A. P., Cami, J., et al. 2012, ApJ, 761, 35

Mizuno, H., Markiewicz, W. J., \& Voelk, H. J. 1988, A\&A, 195, 183

Nakagawa, Y., Nakazawa, K., \& Hayashi, C. 1981, Icarus, 45, 517

Robertson, J. 1986, Adv. Phys., 35, 317

Rouillé, G., Jäger, C., Krasnokutski, S. A., Krebsz, M., \& Henning, T. 2014, Faraday Discussions, accepted

Routly, P. M., \& Spitzer, Jr., L. 1952, ApJ, 115, 227

Seab, C. G. 1987, in Interstellar Processes, eds. D. J. Hollenbach, \& H. A. Thronson, Jr., Astrophys. Space Sci. Lib., 134, 491

Seab, C. G., \& Shull, J. M. 1983, ApJ, 275, 652

Serra Díaz-Cano, L., \& Jones, A. P. 2008, A\&A, 492, 127

Shull, J. M. 1978, ApJ, 226, 858

Smith, R., \& Webb, R. P. 1993, Roy. Soc. London Proc. Ser. A, 441, 495

Snow, T. P., \& Witt, A. N. 1996, ApJ, 468, L65

Tielens, A. G. G. M., McKee, C. F., Seab, C. G., \& Hollenbach, D. J. 1994, ApJ, 431, 321

Weingartner, J. C., \& Draine, B. T. 2001, ApJS, 134, 263

Welty, D. E., Jenkins, E. B., Raymond, J. C., Mallouris, C., \& York, D. G. 2002, ApJ, 579, 304

Ziegler, J. F., Biersack, J. P., \& Littmark, U. 1985, Stopping and Range of Ions in Matter, version 2013, http://www.srim.org

Page 17 is available in the electronic edition of the journal at http://www . aanda. org 


\section{Appendix A: Properties of the skewed Maxwellian distribution}

The skewed Maxwellian distribution, $f_{\text {skM }}$ (see Sect. 3.3), is a velocity probability distribution with integral normalised to unity. In order to prove this property, we integrate over relative velocities, $V_{\text {rel }}$, from $0 \mathrm{~km} \mathrm{~s}^{-1}$ to $+\infty \mathrm{km} \mathrm{s}^{-1}$.

$$
\begin{aligned}
& \int_{0}^{\infty} f_{\mathrm{skM}} v \mathrm{~d} v=\sqrt{\frac{m}{2 k_{\mathrm{B}} T_{\text {gas }} \pi}} \int_{0}^{\infty} \frac{v}{V_{\mathrm{drift}}} \\
& \times\left[\exp \left(-\frac{m}{2 k_{\mathrm{B}} T_{\text {gas }}}\left(v-V_{\mathrm{drift}}\right)^{2}\right)-\exp \left(-\frac{m}{2 k_{\mathrm{B}} T_{\text {gas }}}\left(v+V_{\mathrm{drift}}\right)^{2}\right)\right] \mathrm{d} v .
\end{aligned}
$$

We split the two terms in brackets into two different integrals. In the first term we make the following change of variables $\sqrt{\frac{m}{2 k_{\mathrm{B}} T_{\text {gas }}}}\left(v-V_{\text {drift }}\right)=x$, while in the second term $\sqrt{\frac{m}{2 k_{\mathrm{B}} T_{\text {gas }}}}(v+$ $\left.V_{\text {drift }}\right)=x$. This leads to

$$
\begin{aligned}
\int_{0}^{\infty} f_{\mathrm{skM}} v \mathrm{~d} v & =\sqrt{\frac{m}{2 k_{\mathrm{B}} T_{\text {gas }} \pi}} \frac{1}{V_{\text {drift }}} \\
& {\left[\int_{0}^{\infty}\left(x \sqrt{\frac{2 k_{\mathrm{B}} T_{\text {gas }}}{m}}+V_{\text {drift }}\right) \mathrm{e}^{-x^{2}} \sqrt{\frac{2 k_{\mathrm{B}} T_{\mathrm{gas}}}{m}} \mathrm{~d} x+\right.} \\
& \left.-\int_{0}^{\infty}\left(x \sqrt{\frac{2 k_{\mathrm{B}} T_{\mathrm{gas}}}{m}}-V_{\mathrm{drift}}\right) \mathrm{e}^{-x^{2}} \sqrt{\frac{2 k_{\mathrm{B}} T_{\mathrm{gas}}}{m}} \mathrm{~d} x\right] \\
& =\sqrt{\frac{m}{2 k_{\mathrm{B}} T_{\text {gas }} \pi}} \frac{1}{V_{\text {drift }}} \int_{-\infty}^{\infty} V_{\text {rel }} \sqrt{\frac{2 k_{\mathrm{B}} T_{\mathrm{gas}}}{m} \mathrm{e}^{-x^{2}} \mathrm{~d} x} \\
& =1 .
\end{aligned}
$$

We now demonstrate that the skewed Maxwellian distribution, under the approximation that the thermal motion of the gas ions is negligible with respect to their drift motion, tends to the a Dirac delta probability. In this case we can assume that $T_{\text {gas }} \rightarrow 0$ and that the relative velocity between a grain and the gas equals the drift velocity (i.e. $v \rightarrow V_{\text {drift }}$ ). This then implies

$$
\frac{v}{V_{\text {drift }}} \rightarrow 1
$$

and

$$
\exp \left(-\frac{m}{2 k_{\mathrm{B}} T_{\text {gas }}}\left(v+V_{\text {drift }}\right)^{2}\right) \rightarrow 0 .
$$

Making the following change of variables

$$
\sqrt{\frac{2 k_{\mathrm{B}} T_{\mathrm{gas}}}{m}}=a
$$

the skewed Maxwellian distribution then results

$$
\begin{aligned}
f_{\text {skM }} & =\frac{1}{a \sqrt{\pi}} \exp \left(-\frac{x^{2}}{a^{2}}\right) \\
& =\delta_{a}(x) .
\end{aligned}
$$

As desired, the skewed Maxwellian distribution in this case tends to a Dirac delta probability distribution, i.e. the case for the inertial sputtering described by Tielens et al. (1994), see Sect. 3.3.

\section{Appendix B: Shattering and vapourisation parameters for $\mathrm{a}-\mathrm{C}(\mathrm{H})$ grains}

As shown by Tielens et al. (1994) and Jones et al. (1996), the shattering and vapourisation of colliding grains can be modelled in the framework of shocks in solids. The first step to estimate the relevant parameters involved in the model is to estimate the average binding energy of the target material. Graphitic-type materials consists of purely $\mathrm{sp}^{2}$ hybridised bonds $\mathrm{C}=\mathrm{C}$ while diamond is of purely $\mathrm{sp}^{3} \mathrm{C}-\mathrm{C}$ bonds. An a-C(:H) material is somewhat in between the two materials and present a mix of hybridised $\mathrm{sp}^{3}$ and $\mathrm{sp}^{2}$ bonds with a significant $\mathrm{H}$ atom concentration. The binding energy, $E_{\mathrm{b}}$, for this material can be expressed as

$E_{\mathrm{b}}=E_{\mathrm{C}-\mathrm{C}}+1 / 2 E_{\mathrm{C}=\mathrm{C}}$,

where $E_{\mathrm{C}-\mathrm{C}}(=3.6 \mathrm{eV})$ and $E_{\mathrm{C}=\mathrm{C}}(=6.3 \mathrm{eV})$ are the energy of the $\mathrm{sp}^{3}$ and $\mathrm{sp}^{2}$ bonds, respectively. The a-C $(: \mathrm{H})$ binding energy then yields $E_{\mathrm{b}}=6.5 \mathrm{eV}$.

As discussed by Tielens et al. (1994), the threshold vapourisation energy density, $\epsilon_{\mathrm{v}}$, is related to the binding energy and can be estimated as

$\epsilon_{\mathrm{v}}=\frac{E_{\mathrm{b}}}{m_{\mathrm{p}} M}$,

where $m_{\mathrm{p}}$ is the proton mass and $M$ the carbon atomic mass. Then the minimum relative velocity between the colliding grains for vapourisation (i.e. the threshold vapourisation velocity, $v_{\text {th }}$ ) is

$v_{\text {th }}=2\left(2 \epsilon_{\mathrm{v}}\right)^{1 / 2}$.

For a-C $(: \mathrm{H})$ grains we then obtain a threshold vapourisation energy and velocity of $\epsilon_{\mathrm{v}}=5.2 \times 10^{11} \mathrm{erg} \mathrm{g}^{-1}$ and $v_{\text {th }}=20.3 \times$ $10^{5} \mathrm{~cm} \mathrm{~s}^{-1}$.

At threshold, the pressure of the shock in the solid, $P_{\mathrm{th}, \mathrm{v}}$, can be expressed as

$P_{\mathrm{th}, \mathrm{v}}=\frac{\rho v_{\mathrm{th}}\left(v_{\mathrm{th}}-c_{0}\right)}{s} \times 10^{10} \mathrm{dyne}^{-2}$,

where $c_{0}$ is the speed of sound in the target, $\rho$ its density and $s$ a parameter introduced by McQueen et al. (1970) and estimated to be $s \approx 1.9$ for graphite/a-C materials. We assume that $c_{0} \ll v_{\text {th }}$ and confirm the validity of this assumption below.

In order to estimate the corresponding parameters for the shattering process, we assume that the threshold vapourisation and shattering pressure scale for a-C $(: \mathrm{H})$ materials as for graphite. Jones et al. (1996) made a detailed estimation of these parameters for graphite obtaining $P_{\text {th,v }}=5.8 \times 10^{12}$ dyne $\mathrm{cm}^{-2}$ and $P_{\text {th,sha }}=4.0 \times 10^{10}$ dyne $\mathrm{cm}^{-2}$. Scaling these parameters, for a-C $(: \mathrm{H})$ grains we then have $P_{\text {th,sha }}=2.0 \times 10^{10} \mathrm{dyne}^{-2}$. Furthermore, the threshold shattering pressure is a good estimate of the Young modulus of the material, $E_{\mathrm{Y}}$, and therefore we can calculate the speed of sound in the medium as

$c_{0} \sim \sqrt{\frac{E_{\mathrm{Y}}}{\rho}}$,

obtaining $c_{0} \approx 1$. We finally conclude that the assumption $c_{0} \ll$ $v_{\text {th }}$ is valid in this case. 